\title{
Estrogen-related receptors are targetable ROS sensors
}

\author{
Mathieu Vernier, ${ }^{1}$ Catherine R. Dufour ${ }^{1}$ Shawn McGuirk, ${ }^{1}$ Charlotte Scholtes, ${ }^{1}$ Xiaojing Li, ${ }^{1}$ \\ Guillaume Bourmeau, ${ }^{1}$ Hellen Kuasne, ${ }_{1}$ Morag Park, ${ }^{1,2,3,4}$ Julie St-Pierre, ${ }^{1,2,5}$ Etienne Audet-Walsh, ${ }^{1,6}$ \\ and Vincent Giguère ${ }^{1,2,3,4}$ \\ ${ }^{1}$ Goodman Cancer Research Centre, McGill University, Montréal, Quebec H3A 1A3, Canada; ${ }^{2}$ Department of Biochemistry, \\ ${ }^{3}$ Department of Medicine, ${ }^{4}$ Department of Oncology, McGill University, Montréal, Quebec H3G 1Y6, Canada
}

Excessive reactive oxygen species (ROS) can cause oxidative stress and consequently cell injury contributing to a wide range of diseases. Addressing the critical gaps in our understanding of the adaptive molecular events downstream ROS provocation holds promise for the identification of druggable metabolic vulnerabilities. Here, we unveil a direct molecular link between the activity of two estrogen-related receptor (ERR) isoforms and the control of glutamine utilization and glutathione antioxidant production. ERRa down-regulation restricts glutamine entry into the TCA cycle, while ERR $\gamma$ up-regulation promotes glutamine-driven glutathione production. Notably, we identify increased ERR $\gamma$ expression/activation as a hallmark of oxidative stress triggered by mitochondrial disruption or chemotherapy. Enhanced tumor antioxidant capacity is an underlying feature of human breast cancer (BCa) patients that respond poorly to treatment. We demonstrate that pharmacological inhibition of ERR $\gamma$ with the selective inverse agonist GSK5182 increases antitumor efficacy of the chemotherapeutic paclitaxel on poor outcome BCa tumor organoids. Our findings thus underscore the ERRs as novel redox sensors and effectors of a ROS defense program and highlight the potential therapeutic advantage of exploiting ERR $\gamma$ inhibitors for the treatment of BCa and other diseases where oxidative stress plays a central role.

[Keywords: nuclear receptor; oxidative stress; glutamine; glutathione; breast cancer; chemotherapy; gene signature; metabolic flux; organoid; mitochondria; taxane]

Supplemental material is available for this article.

Received July 23, 2019; revised version accepted January 21, 2020.

Mitochondria are key organelles that coordinate metabolism with cellular functions. One pathway adopted by mitochondria to communicate with the cell is through reactive oxygen species (ROS) signaling. ROS are generated during oxidative phosphorylation where electrons are transported via the electron transport chain (ETC) to create a proton gradient able to oxidize oxygen and create ATP. Eventually, oxygen is directly reduced by the transported electrons, generating ROS. Variations in ROS levels fine-tune numerous signaling pathways via protein modifications to adapt to changes in nutrient and oxidative environment (Shadel and Horvath 2015). Deregulation of mitochondria can result in elevated levels of ROS causing damage to lipids, protein, and DNA, a situation known as oxidative stress, and ultimately to pathophysiological conditions such as diabetes, neurodegeneration,

Present Addresses: ${ }^{5}$ Department of Biochemistry, Microbiology, and Immunology, Ottawa Institute of Systems Biology, Faculty of Medicine, University of Ottawa, Ottawa, Ontario K1H 8M5, Canada; ${ }^{6}$ EndocrinologyNephrology Research Axis, Centre de recherche du CHU de Québec-Université Laval, Department of Molecular Medicine, Faculty of Medicine, Centre de Recherche sur le Cancer de l'Université Laval, Québec City, Quebec G1V 4G2, Canada.

Corresponding author: vincent.giguere@mcgill.ca

Article published online ahead of print. Article and publication date are online at http://www.genesdev.org/cgi/doi/10.1101/gad.330746.119. inflammation, cancer, and aging (Pizzorno 2014). To minimize oxidative damage, cells rely on antioxidant mechanisms that modulate mitochondrial metabolism to maintain cellular homeostasis (Ray et al. 2012). In particular, malignant cells function with higher amounts of ROS than normal cells and ROS have been shown to promote cancer cell proliferation and metastasis (Cairns et al. 2011). To counteract the potential toxic effect of elevated ROS, cancer cells have acquired metabolic alterations that favor key pathways involved in redox homeostasis, such as an increase of the pentose phosphate pathway or glutamine uptake that sustain, respectively, NADPH and glutathione synthesis (Yang et al. 2014; Li et al. 2015).

The estrogen-related receptors (ERRs) $\alpha$ and $\gamma$ are orphan nuclear receptors playing pivotal roles in the control of energy metabolism in both normal and cancerous cells (Giguère 2008; Deblois et al. 2010; Deblois and Giguére 2013; Audet-Walsh and Giguère 2015; Misawa and Inoue 2015; Misra et al. 2017). ERRs, acting as

(C) 2020 Vernier et al. This article is distributed exclusively by Cold Spring Harbor Laboratory Press for the first six months after the full-issue publication date (see http://genesdev.cshlp.org/site/misc/terms.xhtml). After six months, it is available under a Creative Commons License (Attribution-NonCommercial 4.0 International), as described at http://creativecommons.org/licenses/by-nc/4.0/. 
transcriptional modulators of cellular metabolism, regulate the expression of genes involved in glycolysis, fatty acid oxidation, and mitochondrial biogenesis and function including oxidative phosphorylation and electron transport (Mootha et al. 2004; Dufour et al. 2007; Charest-Marcotte et al. 2010; Eichner and Giguère 2011; Chaveroux et al. 2013; Xia et al. 2019). We and others have previously shown that both ERR $a$ and ERR $\gamma$ are implicated in the regulation of ROS signaling (Sonoda et al. 2007; Hong et al. 2013; Murray et al. 2013; Kim et al. 2016; Wu et al. 2016). Notably, ERRa confers resistance to treatment with lapatinib, a dual ErbB2 and epidermal growth factor receptor (EGFR) inhibitor through protection against oxidative stress (Deblois et al. 2016). Also, whereas loss of ERRa blunts ROS provocation by the hepatocellular carcinoma (HCC)-inducing carcinogen diethylnitrosamine $(\mathrm{DEN})$, targeting ERR $\gamma$ in HCC suppresses tumorigenesis in part by inducing ROS (Hong et al. 2013; Kim et al. 2016). While it is known that the ERRs can regulate ROS, the exact mechanisms underlying ERR-dependent ROS regulation remains elusive and the potential functional cross-talk between ERR $\alpha$ and ERR $\gamma$ in ROS homeostasis or whether ROS signaling can modulate the ERRs has not been explored.

In the present study, we reveal that in both normal mouse embryonic fibroblasts (MEFs) and BCa cells, ROS induction leads to a switch in nuclear receptor ERR isoform expression with a noticeable decrease in ERR $\alpha$ and robust augmentation of ERR $\gamma$ protein. Further investigation shows that oxidative stress induced by either the mitochondrial disruptor rotenone or the chemotherapeutic paclitaxel, in fact, activates both transcriptional regulators marked by increased genomic recruitment to cotarget genes. Targeting this ERR-driven adaptive response established their importance for ROS defense due in part via up-regulation of a glutamine/glutathione metabolic gene program. Particularly, inhibition of ERR $\gamma$ with the selective inverse agonist GSK5182 (Chao et al. 2006) sensitized triple-negative $\mathrm{BCa}$ patient-derived xenograft (PDX) organoids to the chemotherapeutic paclitaxel. Our findings support the benefit of antagonizing ERR $\gamma$ activity for cancer disease management by rendering ROS-promoting anticancer agents more effective and may be beneficial to manage a wide range of pathological conditions induced by oxidative stress.

\section{Results}

$E R R \alpha$ and $E R R \gamma$ are redox sensors and regulators of ROS signaling

ROS signaling is one of the principal mechanisms implemented by the mitochondria to coordinate mitochondrial and nuclear functions. Considering the established role of the nuclear receptors ERR $\alpha$ and ERR $\gamma$ as master regulators of mitochondrial activity and our previous finding that ERRa can act as an upstream modifier of the redox state (Eichner and Giguère 2011; Deblois et al. 2016), we first examined whether ROS directly regulates the ERRs. Using MEFs, we first show that the exogenous addition of hydrogen peroxide $\left(\mathrm{H}_{2} \mathrm{O}_{2}\right)$, a natural ROS produced by mitochondria, leads to the simultaneous diminution of ERR $\alpha$ and accumulation of ERR $\gamma$ in a concentration- and time-dependent manner (Fig. 1A,B). Boosting intracellular mitochondrial ROS generation via disruption of normal mitochondrial function by addition of the electron transport chain (ETC) inhibitors rotenone, thenoyltrifluoroacetone (TTFA), or oligomycin targeting complex I, II, or IV, respectively, also noticeably reduced ERR $\alpha$ protein while inducing ERR $\gamma$ levels (Fig. 1C).

Next, we investigated whether the inverse effect of ROS signaling on ERR $\alpha$ and ERR $\gamma$ protein observed in MEFs extends to that of human cancer cells. To this end, we used two established BCa cell lines, BT474 and SKBR3, representing Luminal B $\left(\mathrm{ER}^{+}, \mathrm{HER} 2^{+}\right)$and HER2 ${ }^{+}\left(\mathrm{ER}^{-}\right)$molecular subtypes, respectively. Indeed, treatment of $\mathrm{BCa}$ cells with either $\mathrm{H}_{2} \mathrm{O}_{2}$ or inducers of mitochondrial dysfunction lowered ERRa levels on one hand while stimulating ERR $\gamma$ levels on the other (Fig. 1D-F). Notably, the rotenone-induced ERR isoform switch was reversed by pretreatment of SKBR3 cells with the ROS scavenger MitoQ, denoting that the ERRs are downstream targets of ROS signaling (Fig. 1G). Addition of the proteasome inhibitor MG132 was able to rescue the 1-h rotenone-mediated degradation of ERR $\alpha$ in SKBR3 cells, a treatment time that had no significant impact on the transcript levels of ERRa (Esrra) and ERR $\gamma$ (Esrrg) (Fig. 1H,I), suggesting that modulation of ERR levels by ROS signaling occurs initially at the protein, not transcriptional level. A longer exposure to rotenone for $6 \mathrm{~h}$ ultimately impacted ERR $\alpha$ and ERR $\gamma$ mRNA levels with an observed up-regulation of Esrrg and down-regulation of Esrra (Supplemental Fig. S1A). To establish a functional cross-talk between ROS and the ERRs, we next assessed the impact of ERRa and ERR $\gamma$ inhibitors on rotenone-provoked ROS levels (Supplemental Fig. S1B). As a proof of concept, rotenone treatment alone increased ROS levels of SKBR3 cells as determined by the elevated production of the oxidized fluorescent probe $2^{\prime}, 7^{\prime}$-dichlorofluorescein (DCF) from $2^{\prime}, 7^{\prime}$-dichlorodihydrofluorescein diacetate $\left(\mathrm{H}_{2} \mathrm{DCFDA}\right)$ by ROS, signified by a shift of the DCF fluorescent peak to the right (Fig. 1J; Supplemental Fig. S1C). While ERR $\alpha$ inhibition by the selective inverse agonist compound 29 (C29) (Patch et al. 2011) decreased rotenone-stimulated ROS, displayed by a shift of the rotenone-produced DCF peak to the left, abrogating ERR $\gamma$ activity by GSK5182 resulted in an overall higher level of ROS production (Fig. 1J; Supplemental Fig. S1C).

We next questioned whether the ERRs can alter ROS levels in the absence of an inducer of oxidative stress. Remarkably, MEFs lacking ERR $\alpha(\mathrm{KO})$ exhibited diminished ROS levels in the basal state and re-expression of ERRa in KO MEFs elevated ROS production (Fig. 2A; Supplemental Fig. S2). siRNA-mediated knockdown of ERR $\gamma$ in ERR $\alpha$ KO MEFs, found to express higher levels of ERR $\gamma$ compared with WT MEFs, robustly augmented ROS (Fig. 2A; Supplemental Fig. S2). Furthermore, pharmacological inhibition of ERR $a$ with C29 in SKBR3 cells was proven effective in decreasing cellular ROS levels, an effect that was impaired by cotreatment with the ERR $\gamma$ inhibitor GSK5182 (Fig. 2B; 
Vernier et al.

A

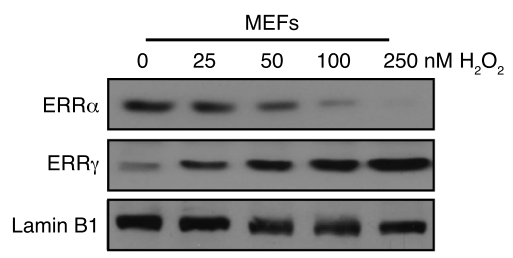

D

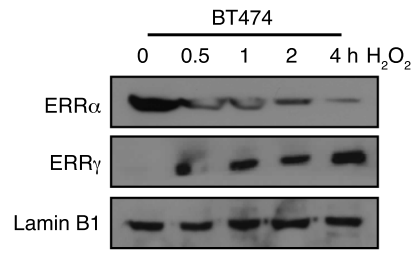

G

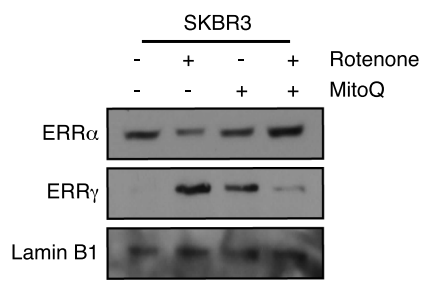

$\mathbf{J}$

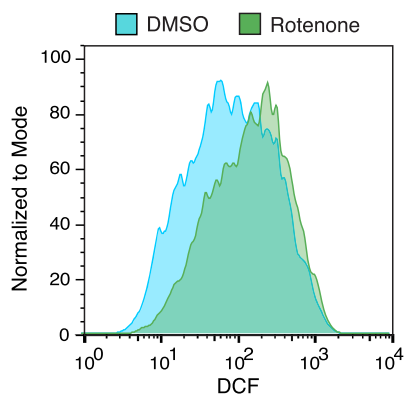

B

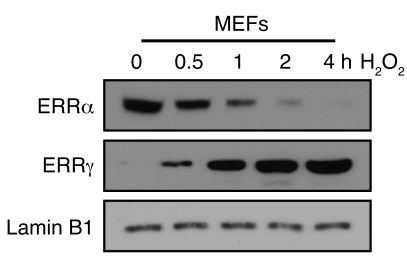

$\mathbf{E}$

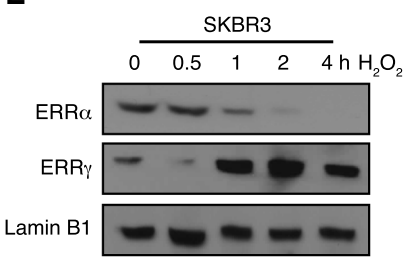

H
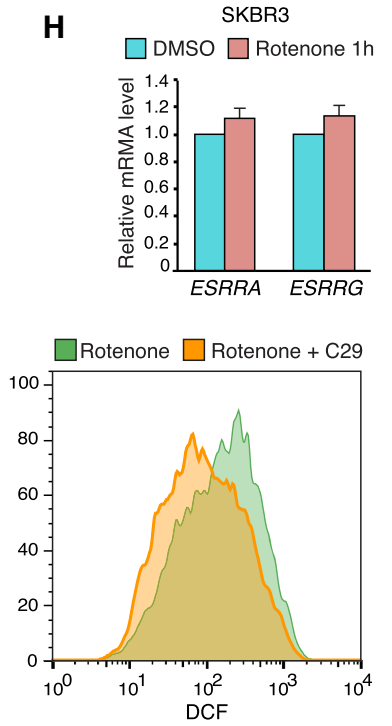

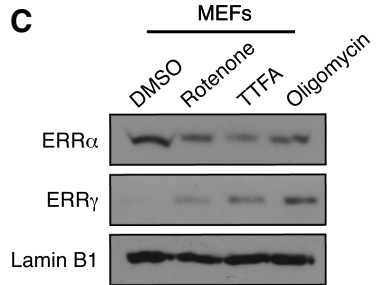

$\mathbf{F}$

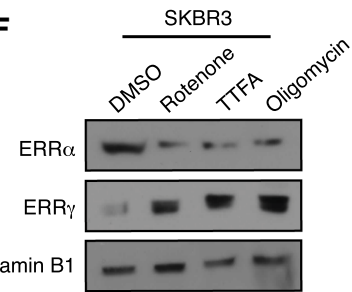

I

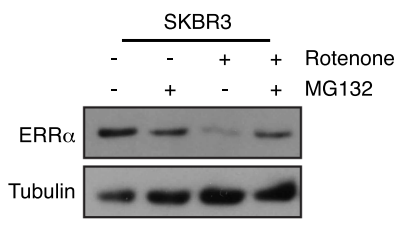

Figure 1. Cross-talk between ROS signaling and the ERRs. $(A, B)$ ERR $\alpha$ and ERR $\gamma$ protein determination in MEFs after a 1-h treatment with increasing concentrations of hydrogen peroxide $\left(\mathrm{H}_{2} \mathrm{O}_{2}\right)$ as indicated $(A)$ or a time course following treatment with $150 \mu M \mathrm{H}_{2} \mathrm{O}_{2}$ $(B)$. (C) ERR isoform immunoblotting in MEFs following treatment with $1 \mu \mathrm{M}$ of the mitochondrial disruptors, rotenone, thenoyltrifluoroacetone (TTFA), or oligomycin for $1 \mathrm{~h}$. $(D, E)$ Time-dependent effect of $150 \mu \mathrm{M} \mathrm{H}_{2} \mathrm{O}_{2}$ on ERR $\alpha$ and ERR $\gamma$ expression in BT474 (D) and SKBR3 $(E)$ cells. $(F)$ Effect of a 1-h treatment with $1 \mu \mathrm{M}$ rotenone, TTFA, or oligomycin on ERR $\alpha$ and ERR $\gamma$ protein levels in SKBR3 cells. $(G)$ Reversal of rotenone-induced ERR a degradation and ERR $\gamma$ stabilization in SKBR3 cells by a 1-h pretreatment with the ROS scavenger mitoQ $(1 \mu \mathrm{M})$. (H) Transcript levels of the ERR $\alpha$ and ERR $\gamma$-encoding genes, ESRRA and ESRRG, respectively, following a 1-h treatment with rotenone. (I) Rotenone-induced ERRa degradation following a 1-h treatment was rescued by a pretreatment with the proteasome inhibitor MG132 for $2 \mathrm{~h}(10 \mathrm{nM})$. (J) Measurement of ROS levels by fluorescence of oxidized 2', $7^{\prime}$-dichlorofluorescein (DCF) in SKBR3 cells treated with the ROS-inducing drug rotenone alone or in combination with an inhibitor of ERRa (C29) or ERR $($ GSK5182). Data in $H$ represent mean \pm SEM.

Supplemental Fig. S2). Excessive ROS can lead to the oxidative damage of macromolecules, in particular nucleic acids (Cadet and Wagner 2013); thus, we next evaluated ROS-induced DNA damage in response to modulation of ERR $\alpha$ and ERR $\gamma$ activity. Concomitant with the observed reduction of ROS upon ERRa inhibition, the levels of phosphorylated histone $\mathrm{H} 2 \mathrm{AX}(\gamma \mathrm{H} 2 \mathrm{AX})$, a well-known marker of DNA damage, and immunofluorescence staining of oxidized guanine, 8-oxoguanine, were significantly decreased in both BT474 and SKBR3 cells following C29 treatment (Fig. 2C,D). Importantly, this effect was ERR $\gamma$ - dependent as coadministration of the ERR $\gamma$ inhibitor GSK5182 derepressed and generally restored the magnitude of DNA damage in the presence of C29 (Fig. 2C,D). The data support the existence of a functional cross-talk between ROS signaling and the ERRs.

\section{$E R R \alpha$ and ERR $\gamma$ cotarget a ROS homeostasis transcriptional network}

To help delineate mechanistically how perturbing ERRa and $E R R \gamma$ activity can function as a ROS defense response, 

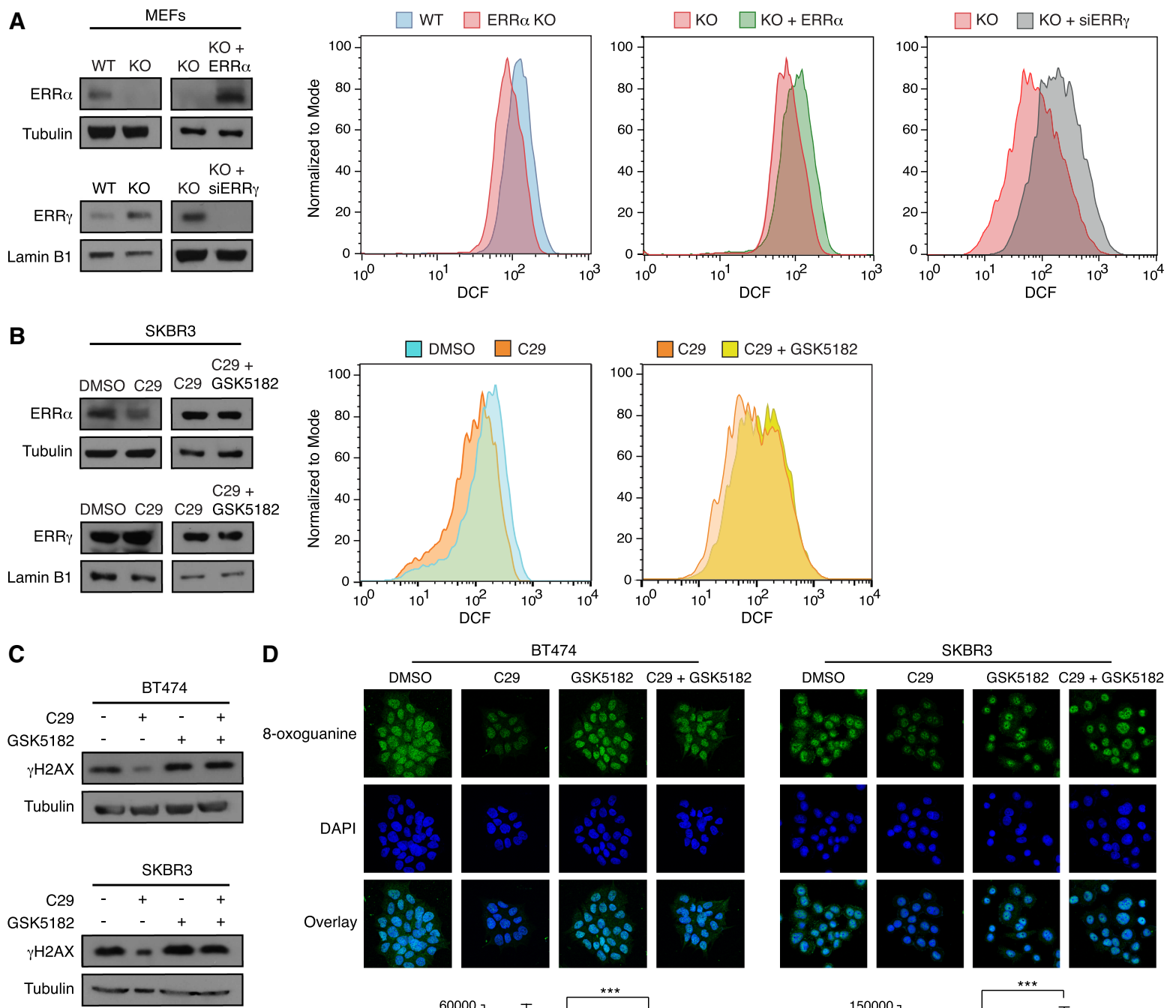

D
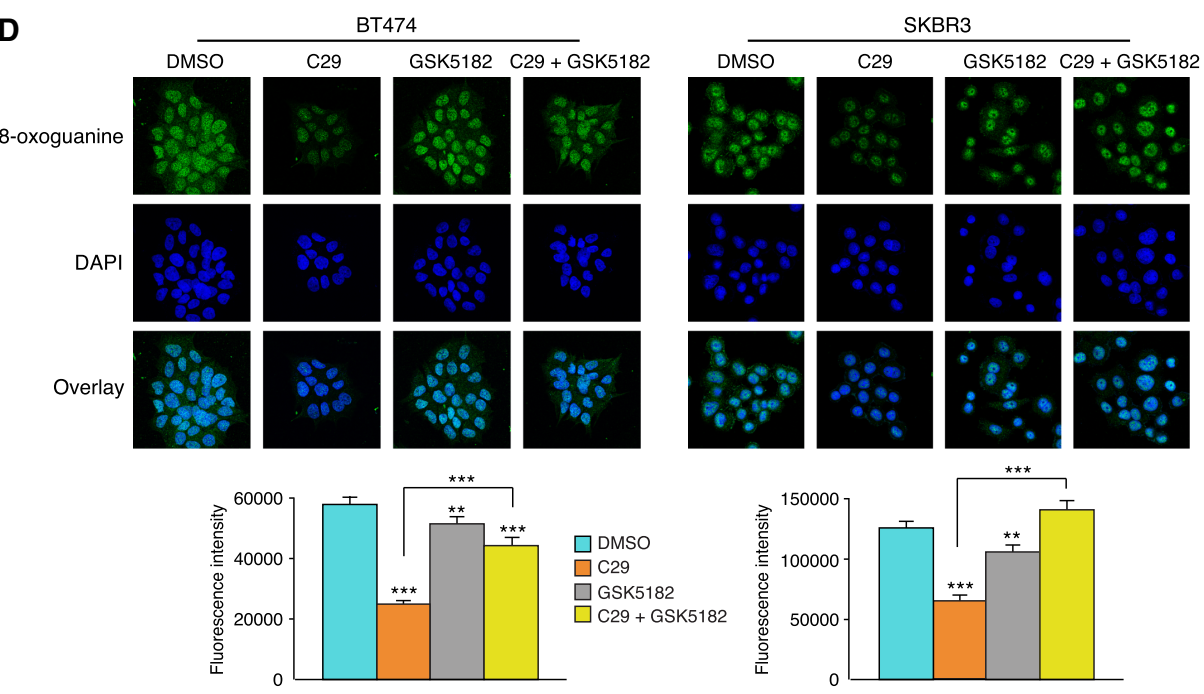

Figure 2. ERRa and ERR $\gamma$ differentially modulate ROS signaling. $(A)$ Measurement of ROS levels by fluorescence of oxidized 2', $7^{\prime}$ dichlorofluorescein (DCF) in WT versus ERRa-null (KO) MEFs as well as in KO versus KO MEFs either re-expressing ERRa or transfected with specific siRNAs against ERR $\gamma(\operatorname{siERR} \gamma)$ for $48 \mathrm{~h}$. (B) The reduction in ROS determined by fluorescence of oxidized DCF following a 24-h treatment of SKBR3 cells with the ERRa inhibitor C29 was found abrogated by cotreatment with the specific ERR $\gamma$ inhibitor GSK5182. $(C)$ Levels of the DNA damage marker, $\gamma \mathrm{H} 2 \mathrm{AX}$, in BCa cells following treatment with ERR a and/or ERR $\gamma$ inhibitors, C29, and/or GSK5182, respectively. (D) Fluorescence detection and quantification of 8-oxoguanine DNA damage in BT474 and SKBR3 cells upon ERR $a$ inhibition with C29 in the presence or absence of the ERR $\gamma$ inhibitor GSK5182. $(A-D)$ Three independent experiments were performed with two replicates per condition and one representative experiment is shown. Data represent mean \pm SEM. $\left(^{* *} \mid P<\right.$ $0.01 ;\left(^{* * *}\right) P<0.001$, Student's $t$-test.

we pursued the identification of an ERR-dependent gene network implicated in this process given the nature of their roles as transcriptional regulators. First, we manually compiled a list of 416 genes taken from distinct metabolic KEGG pathways associated with ROS homeostasis that included glutamine/glutathione metabolism, glycolysis, fatty acid oxidation (FAO), and the TCA cycle/ETC (Fig. 3A; Supplemental Table S1; Panieri and Santoro 2016). As glutamine is a key substrate for glutathione pro- duction, a major cellular antioxidant capable of mitigating ROS damaging effects, we expanded this list to include 14 known genes encoding glutamine transporters (Bhutia and Ganapathy 2016) as they were not among the KEGG pathways used, resulting in a 430-gene set related to ROS homeostasis (Supplemental Table S1). Second, we performed ERR $\gamma$ chromatin immunoprecipitation (ChIP) assays coupled with DNA sequencing (ChIP-seq) in the human BCa cell line BT474, as we determined previously 
Vernier et al.

A
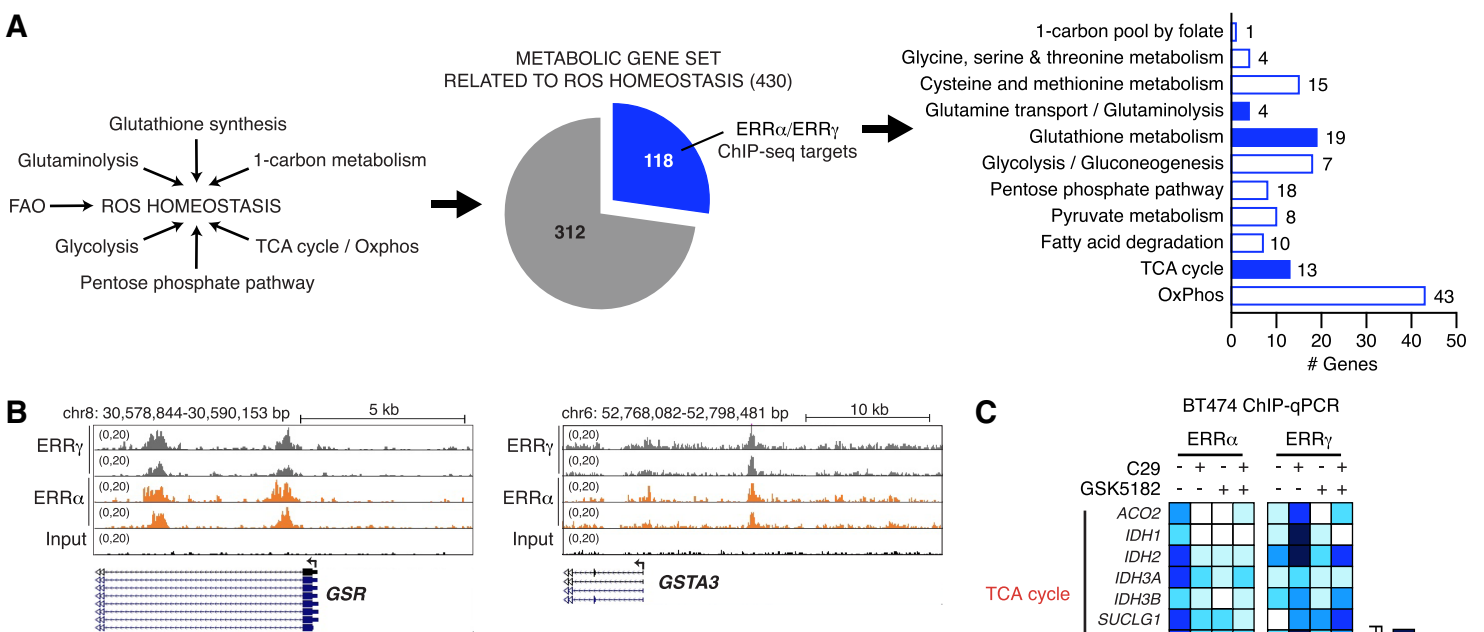

C
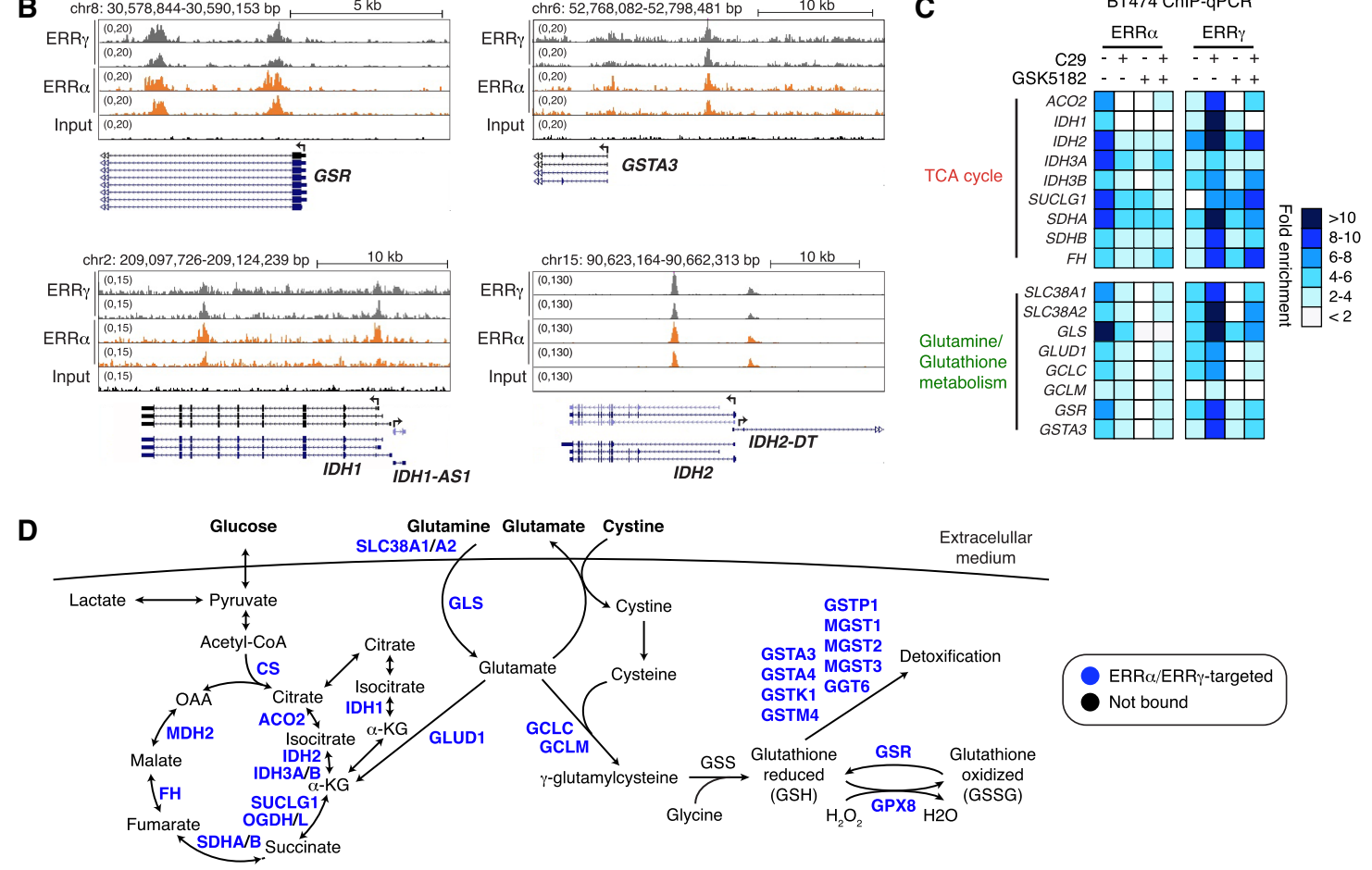

E
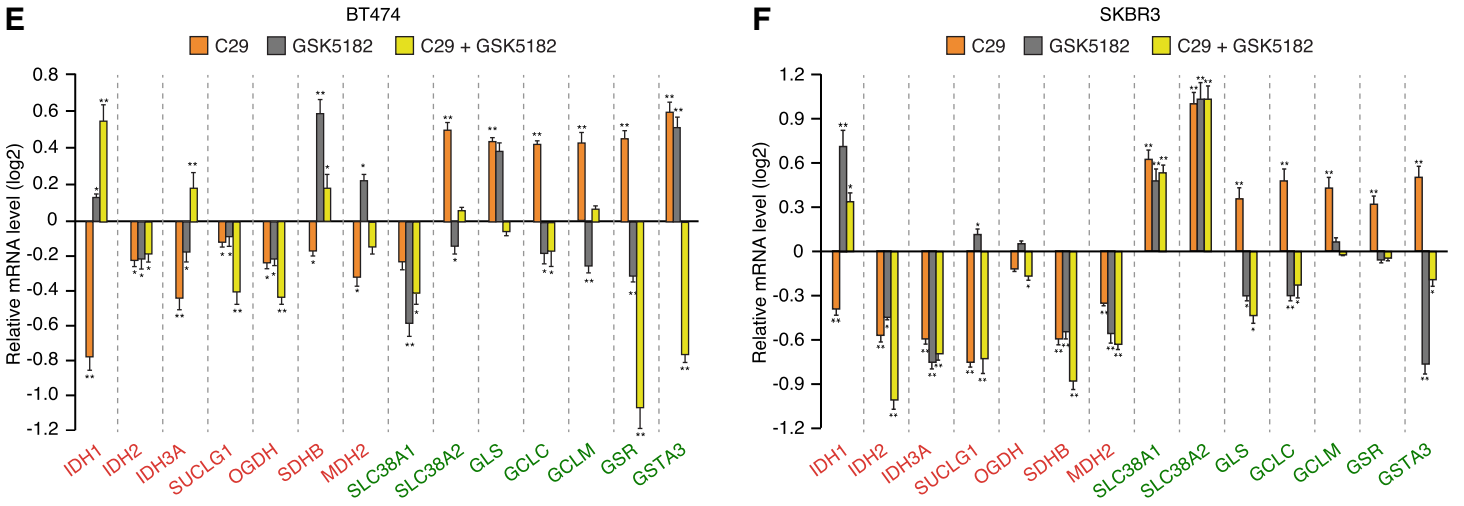

Figure 3. ERR $a$ and ERR $\gamma$ cotarget an ROS homeostasis gene signature. (A, left panel) Schematic illustrating the metabolic pathways that impact ROS homeostasis. (Middle panel) Pie chart shows that 118 of 430 genes of a curated metabolic gene set related to ROS homeostasis are cobound by ERR $\alpha$ and ERR $\gamma$ via intersection of a previous ERR $\alpha$ ChIP-seq study (GSE75876) with that of ERR $\gamma$ in BT474 cells provided here. Only genes with ERR binding $\pm 20 \mathrm{~kb}$ of the TSS of genes were considered. (Right panel) Association of the 118 cotargeted ERR $/ \gamma$ gene set to specific ROS-related metabolic pathways are shown. (B) Genome browser views of ChIP-seq binding events in BT474 cells showing cotargeting of ERRa and ERR $\gamma$ to several genes identified in $A$. $(C)$ Standard ChIP-qPCR analyses in BT474 cells showing the effect of specific ERR $\alpha$ and ERR $\gamma$ inhibitors, C29 and GSK5182, respectively, on the binding of ERR $\alpha$ and ERR $\gamma$ to TCA cycle and glutamine/ glutathione metabolism target genes identified in $A$. (D) Schematic representation of a subset of the ERR $\alpha / \gamma$ targeted ROS homeostasis gene signature identified in $A$ with a central focus on glutamine metabolism toward glutathione generation and its interconnection with the TCA cycle. $(E, F)$ qRT-PCR analysis of a subset of validated ERRa/ $\gamma$ TCA cycle and glutamine/glutathione target genes in $C$ following treatment with C29 or GSK5182 in both BT474 $(E)$ and SKBR3 $(F)$ cells. Data represent mean \pm SEM. $\left({ }^{*}\right) P<0.05 ;\left({ }^{* *}\right) P<0.01$, Student's $t$-test. 
the ERRa cistrome in these cells (Supplemental Table S2; Audet-Walsh et al. 2016). ERR $\gamma$ shares high sequence homology with ERRa, particularly in the DNA-binding domain, and we showed previously that they are corecruited to a large set of genes (Dufour et al. 2007). Not surprisingly, ERR $\gamma$ exhibited a genome location-based distribution in binding peaks like that previously observed for ERRa (Supplemental Fig. S3A; Audet-Walsh et al. 2016). While a larger number of binding sites were identified for ERRa compared with ERR $\gamma(22,538$ vs. 15,223), comparison of the ChIP-seq data sets revealed $>80 \%$ overlap in their binding peaks $(12,445)$ relative to the number of ERR $\gamma$-bound regions (Supplemental Fig. S3B). De novo motif enrichment analysis identified the ERR response element (ERRE), FOXA1 and AP2 as the top three significantly enriched motifs for both ERRa and ERR $\gamma$ ChIP-seq data sets (Supplemental Fig. S3B,C). Finally, we intersected our curated metabolic ROS homeostasis gene set (430) with the list of ChIP-seq ERRa/ $\gamma$ cotargeted genes displaying an overlap in binding events within \pm 20 $\mathrm{kb}$ from the transcriptional start site (TSS) of genes. Our analysis resulted in the identification of $118 \mathrm{ERRa} / \gamma$ target genes implicated in multiple biological processes related to ROS homeostasis of which we focused our attention on those directly impacting glutamine fate, being glutamine/glutathione metabolism and the TCA cycle (Fig. 3A).

Glutamine metabolism drives glutathione production, and once incorporated into the cell, is transformed into glutamate which apart from opting for an anaplerotic route via entry into the TCA cycle by its oxidative deamination to $a$-ketoglutarate $(\alpha-K G)$, glutamate can alternatively be condensed with cysteine to form the precursor to glutathione synthesis, $\gamma$-glutamylcysteine. Cysteine can arise either from the methionine cycle through the transsulfuration pathway or be absorbed in the form of cystine from the extracellular medium via the cystine/glutamate antiporter. ERRa and ERR $\gamma$ ChIP-seq genome browser views and standard ChIP-qPCR validation of a subset of the identified glutamine/glutathione and TCA cycle target genes are shown (Fig. 3B-D; Supplemental Fig. S3D). Several relevant genes, $F H, S D H A, S D H B$, and GCLM, were also validated by ChIP-qPCR as targets of both factors despite the missed calling of significant peaks from the ERR $\gamma$ ChIP-seq profiles (Fig. 3C,D; Supplemental Fig. $\mathrm{S} 3 \mathrm{D})$, enlarging the identified ERRa/ $\gamma$ cotargeted ROS homeostasis-related gene program from 118 to 122 genes (Supplemental Table S3). Treatment of cells with the ERRa inhibitor C29 markedly suppressed ERRa gene occupancy and enhanced ERR $\gamma$ recruitment to the same loci (Fig. 3C). Inactivation of ERR $\gamma$ by GSK5182 generally reduced its binding to DNA and impaired the increased genomic recruitment of ERR $\gamma$ in response to ERRa inhibition by C29 (Fig. 3C). Intriguingly, we found that GSK5182 also impeded ERRa binding to target genes (Fig. 3C), indicating that the presence of ERR $\gamma$ on DNA may be necessary for ERR $\alpha$ recruitment to certain genes. Gene expression analysis in BT474 and SKBR3 cells showed that treatment with either C29 or GSK5182 alone can decrease the mRNA levels of several TCA cycle en- zymes (Fig. 3E,F). Given that the ERRa inhibitor C29 increases ERR $\gamma$ binding to these genes and that the ERR $\gamma$ inhibitor GSK5182 impairs the binding of both factors, this suggests that loss of ERRa recruitment to these genes plays a dominant underlying role for the observed transcriptional repression. One exception among the TCA cycle enzymes tested and found conserved between BT474 and SKBR3 cells was the inverse regulation of the isocitrate dehydrogenase 1 gene, IDH1, by the ERRs (Fig. 3E, F). Reductive carboxylation by which $\alpha-\mathrm{KG}$ is converted to isocitrate prior to citrate formation can be catalyzed by either IDH1 or IDH2, localized in the cytoplasm or mitochondria, respectively (Fig. 3D). The fact that ERR $\alpha$ positively and ERR $\gamma$ negatively regulated $I D H 1$ transcription suggests that the ERRs may play opposing roles on reductive carboxylation. Further expression profiling discovered that C29-mediated ERRa inhibition boosts transcription of glutamine/glutathione pathway genes GLS, GCLC, GCLM, GSR, and GSTA3 in both cell lines tested (Fig. $3 \mathrm{E}, \mathrm{F})$. It is noteworthy that GCLC and GCLM encode the two subunits forming the heterodimeric enzyme glutamate-cysteine ligase (GCL) responsible for the first and rate-limiting step of glutathione synthesis and the glutathione-disulfide reductase-encoding gene GSR helps maintain glutathione availability for detoxification by reverting its oxidized form to a reduced state (Fig. 3D). Strikingly, the C29-driven induction of these genes was found dependent on ERR $\gamma$ as cotreatment with the ERR $\gamma$ inhibitor GSK5182 either abolished or reversed this effect (Fig. $3 \mathrm{E}, \mathrm{F})$. These results imply that ERR $\gamma$ up-regulation in the presence of C29 is largely responsible for the observed transcriptional activation of the glutamine/glutathione pathway. Overall, functional genomics analysis reinforces the existence of an operative functional link between the ERRs and the control of ROS homeostasis.

\section{Modulation of ERR $\alpha$ and ERR $\gamma$ activity perturbs glutamine metabolic flux}

Inhibition of either ERR $\alpha$ or ERR $\gamma$ was found to alter the binding of both receptors to key genes involved in the TCA cycle, glutamine oxidative metabolism and glutathione production, as such we proceeded to determine the functional consequence of ERR $\alpha$ and/or ERR $\gamma$ loss of function on these biological processes. Previously, we showed that C29-mediated ERRa inhibition restricts glutamine flux into the TCA cycle as determined by stable isotoperesolved metabolomics (SIRM) experiments (Deblois et al. 2016). These findings were replicated in this study by SIRM with glutamine $\left(\left[\mathrm{U}-{ }^{13} \mathrm{C}\right]\right.$-glutamine $)$ as the tracer in SKBR3 cells, confirming that inhibiting ERRa function suppresses glutamine-derived $\alpha-K G$ and its further oxidation in the forward direction of the TCA cycle as well as its conversion to citrate in the reverse direction by reductive carboxylation (Fig. 4A). Inhibition of ERR $\gamma$ by GSK5182 had no significant effect on glutamine flux in the forward (oxidative) TCA cycle which remained down-regulated in the presence of C29 (Fig. 4A). However, contrary to ERRa inhibition, ERR $\gamma$ inactivation up-regulated glutamine-driven TCA cycle reductive 
Vernier et al.

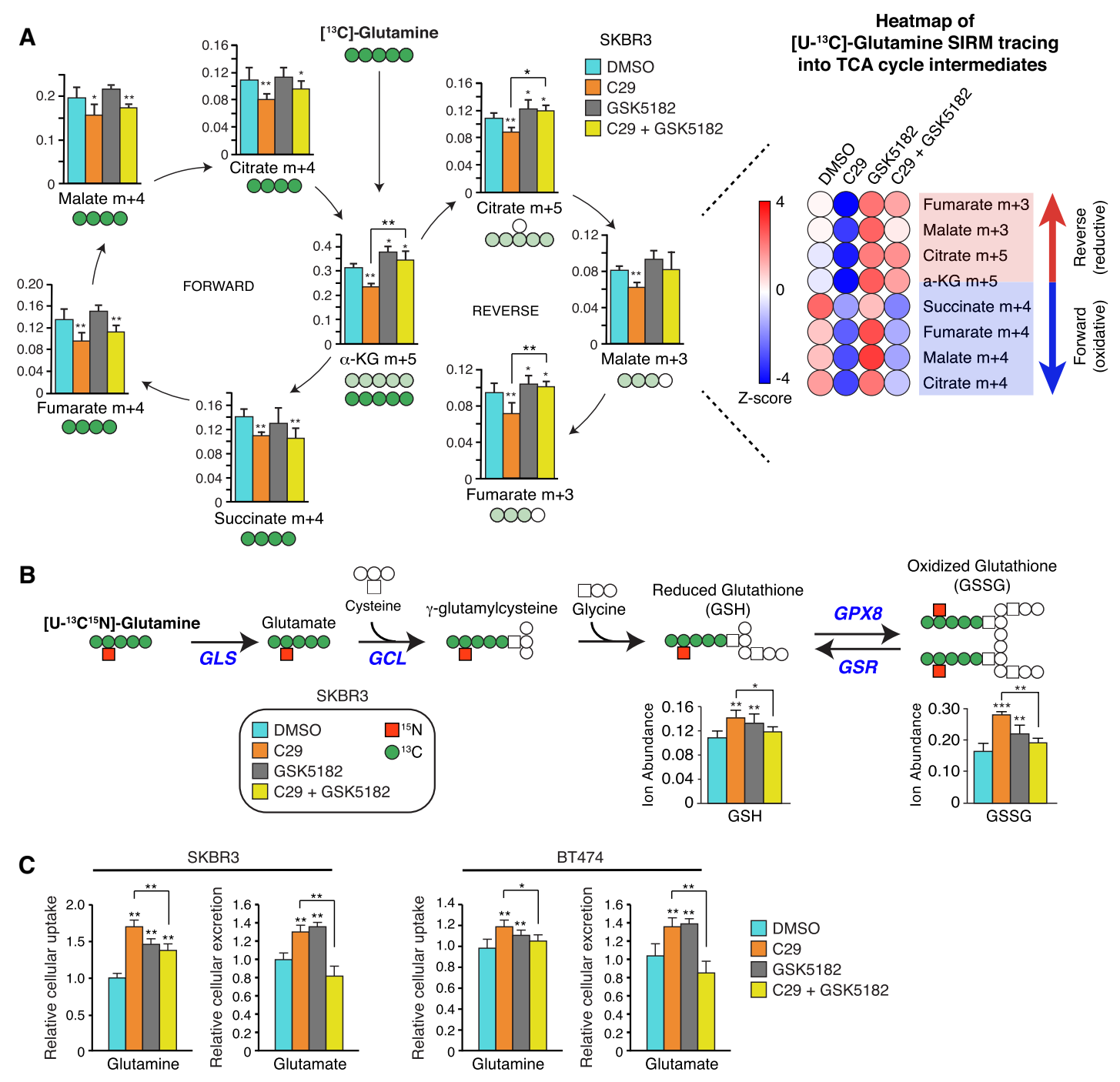

Figure 4. The activities of ERR $\alpha$ and ERR $\gamma$ dictate glutamine fate. $(A)$ SIRM experiments showing labeled glutamine $\left(\left[\mathrm{U}^{-13} \mathrm{C}\right]\right.$-glutamine $)$ tracing measurements into TCA cycle intermediates in both forward and reverse directions in SKBR3 cells treated with vehicle, the ERR $\alpha$ inhibitor C29, and/or the ERR $\gamma$ inhibitor GSK5182. The data represents an average of two independent experiments with five replicates per condition. A $z$-scored heat map representation of the relative SIRM tracing measurements is shown for added clarity. $(B)$ Similar to $A$, SIRM tracing with labeled glutamine $\left(\left[\mathrm{U}_{-}{ }^{13} \mathrm{C}^{15} \mathrm{~N}\right]\right.$-glutamine) to de novo glutathione production was performed in SKBR3 cells treated with or without ERR $\alpha$ and ERR $\gamma$ inhibitors. $(C)$ Effect of ERR $\alpha$ and/or ERR $\gamma$ inhibitors on the relative abundance of glutamine and glutamate in the extracellular media of both SKBR3 and BT474 cells. Data represent mean \pm SEM. $\left({ }^{*}\right) P<0.05 ;\left({ }^{* *}\right) P<0.01 ;\left({ }^{* * *}\right) P<0.001$, Student's $t$-test.

carboxylation, a dominant phenotype in cells cotreated with C29 and GSK5182 (Fig. 4A). The observed effects of ERR $\alpha$ and/or ERR $\gamma$ inhibition on glutamine entry into the TCA cycle are supported by the down-regulation of TCA cycle gene expression by C29 and up-regulation of IDH1 expression by GSK5182 important for reductive carboxylation (Fig. 3E,F).

Next, we assessed the importance of the ERRs on glutamine-driven glutathione production. First, measurement of steady state reduced (GSH) and oxidized (GSSG) glutathione in SKBR3 cells showed reduced GSH and higher GSSG levels in cells treated with either C29 or GSK5182 alone (Supplemental Fig. S4A). Importantly, addition of GSK5182 abolished the observed C29-dependent effects (Supplemental Fig. S4A). Next, using SIRM to follow the flux of glutamine $\left(\left[\mathrm{U}-{ }^{13} \mathrm{C}^{15} \mathrm{~N}\right]\right.$-glutamine) towards de novo glutathione production in SKBR3 cells revealed differential results compared with steady-state levels. Notably, inhibition of ERR $\alpha$ by C29 in SKBR3 cells significantly increased GSH and GSSG generation from glutamine as the tracer, an effect that was abolished by cotreatment with the ERR $\gamma$ inhibitor GSK5182 (Fig. 4B; Supplemental Fig. S4B). In line with these results, attenuation of ERR $\gamma$ activity impaired the C29-dependent increase in both glutamine uptake and extracellular glutamate levels (Fig. 4C), the latter being an indicator of exchange of glutamate for cystine needed for glutathione synthesis (Fig. 3D). Unexpectedly, however, we found 
that GSK5182-mediated ERR $\gamma$ inhibition alone can stimulate glutamine uptake, glutamate excretion, and glutathione generation (Fig. 4B,C). These findings contrast with GSK5182-mediated down-regulation of glutamine/ glutathione gene expression (Fig. 3E,F), suggesting that other factors underlie the observed GSK5182-stimulated glutathione synthesis. Nevertheless, our findings that ERR $\gamma$ promotes ROS formation (Figs. 1, 2), and that glutamine-driven glutathione production upon ERRa loss of function is contingent on ERR $\gamma$ activity supports the key role of ERR $\gamma$ as a defender against ROS.

\section{$E R R \gamma$ loss of function increases rotenone-induced cytotoxicity}

While we observed that perturbing ERR isoform function influences ROS and DNA damage levels in the basal state (Fig. 2), siRNA-mediated silencing or drug-induced inhibition of ERR a or ERR $\gamma$ in SKBR3 cells had no consequence on cellular viability (Fig. 5A,B). However, under conditions of oxidative stress provoked by the mitochondrial disruptor rotenone, which we found to induce $\operatorname{ERR} \gamma$ (Fig. 1), abrogation of ERR $\gamma$ potentiated rotenone-induced cell death (Fig. 5A,B). ERRa loss of function had no impact on the cytotoxic effects of rotenone (Fig. 5A,B), as opposed to ERRa overexpression, which heightened rotenone-induced cell death (Supplemental Fig. S5). To provide mechanistic in- sight into these observations, we assessed ERRa and ERR $\gamma$ transcriptional function in the presence of rotenone. Surprisingly, although acute treatment with rotenone diminishes ERRa protein and augments ERR $\gamma$ expression (Fig. 1), rotenone promoted the recruitment of both factors to cotarget genes, an effect found more striking for ERR $\gamma$ (Fig. 5C). These results thus indicate that both ERRa and ERR $\gamma$ are activated in response to oxidative stress, and treatment of cells with either C29 or GSK5182 was sufficient to block or reverse rotenone-induced up-regulation of several TCA cycle and glutamine/glutathione pathway genes (Fig. 5D). These results diverge from normal conditions where C29-mediated ERRa inhibition leads to upregulation of glutamine/glutathione gene expression due to increased ERR $\gamma$ target gene occupancy (Fig. 3). Here, under conditions of oxidative stress, C29 treatment could not boost ERR $\gamma$ DNA binding but rather had the inverse effect and compromised rotenone-induced ERR $\gamma$ binding and expression of glutamine/glutathione genes (Fig. 5C,D). It is conceivable to think that ERR $\alpha / E R R ~ \gamma$ heterodimerization on chromatin in a state of oxidative stress is necessary for transcriptional control by the ERRs. Taken together, our results indicate that the ERRs play important roles in ROS homeostasis under both normal and oxidative stress conditions and particularly highlight ERR $\gamma$ up-regulation as a critical node in the transcriptional control of glutathione production.

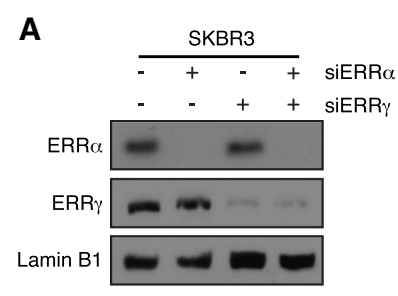

C

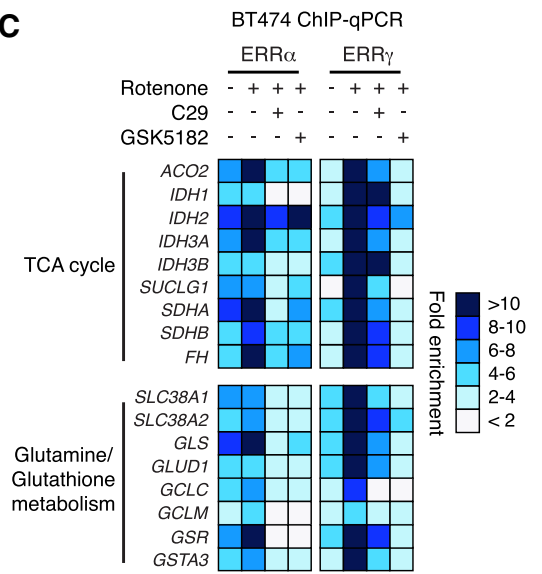

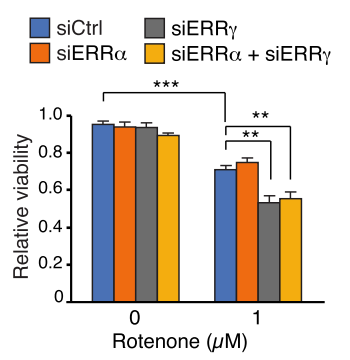

D

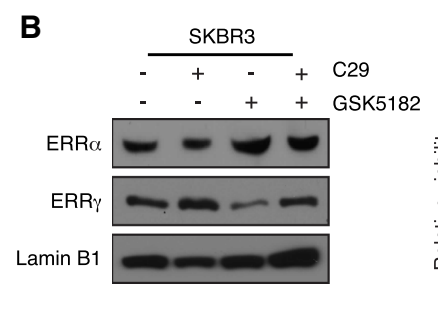

BT474
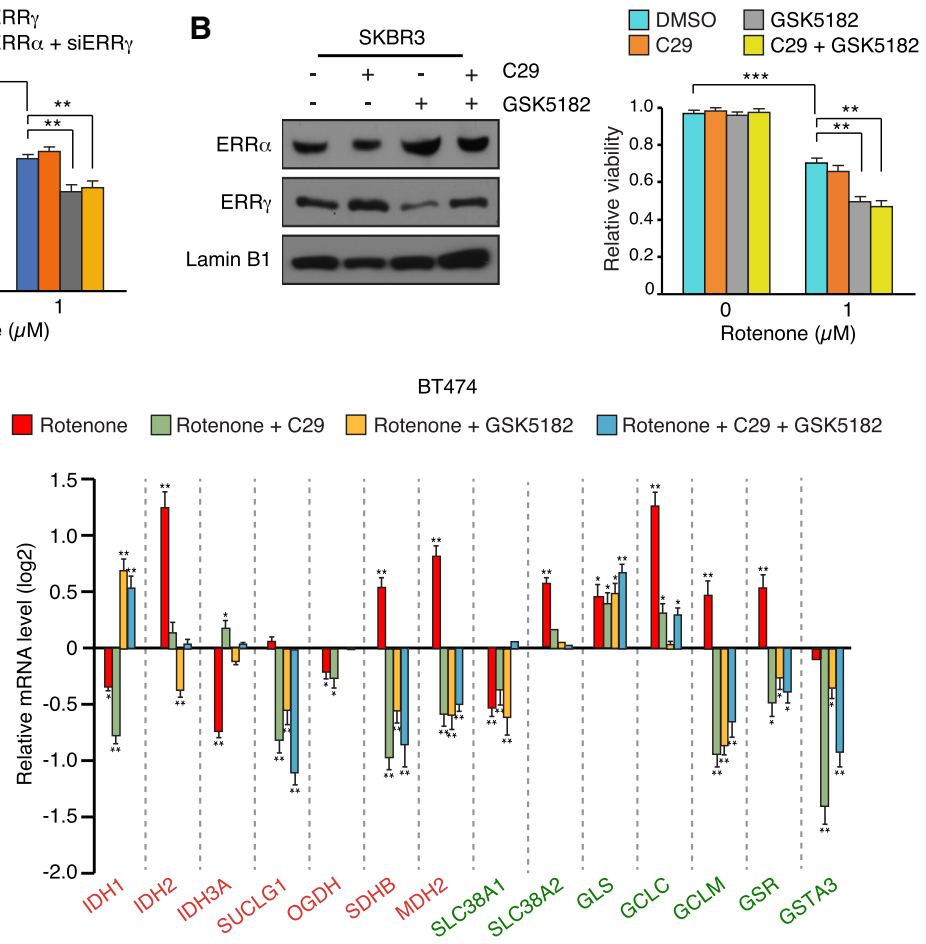

Figure 5. ERR $\gamma$ inhibition increases rotenone-induced cytotoxicity. $(A, B)$ siRNA-mediated knockdown $(A)$ or pharmacological inhibition of ERR $\gamma$ by GSK5182 (B) sensitizes SKBR3 cells to rotenone-induced cell death, while ERRa knockdown or inhibition by C29 has no effect. (C) Standard ChIP-qPCR analyses in BT474 cells showing the effect of a 1-h rotenone treatment on ERR $\alpha$ and ERR $\gamma$ binding to TCA cycle and glutamine/glutathione metabolism cotarget genes with or without pretreatment with C29 or GSK5182. (D) qRTPCR analysis of a subset of TCA cycle and glutamine/glutathione target genes upon treatment with rotenone \pm C29 or GSK5182 in BT474 cells. Data represent mean \pm SEM. $\left({ }^{*}\right) P<0.05 ;\left(^{* *}\right) P<0.01 ;\left(^{* * *}\right) P<0.001$, Student's $t$-test. 
The selective ERR $y$ inhibitor GSK5182 sensitizes BCa cells to chemotherapy

As the data implicate ERR $\gamma$ up-regulation as an important adaptive response to oxidative stress, this makes ERR $\gamma$ an attractive druggable target that may work to sensitize cancer cells to ROS-based chemotherapy. Notably, treatment of both BT474 and SKBR3 cells with three commonly used chemotherapeutics in the clinic for the treatment of $\mathrm{BCa}$, paclitaxel, docetaxel, and doxorubicin, all amplified ERR $\gamma$ levels while reducing ERRa expression (Fig. 5A-C). Forced expression of ERRa had no impact on paclitaxel-induced ERR $\gamma$ expression (Supplemental Fig. S6A). As observed with rotenone, paclitaxel stimulated both ERRa and ERR $\gamma$ target gene recruitment (Fig. 6D), reinforcing that both receptors are activated in response to oxidative
A
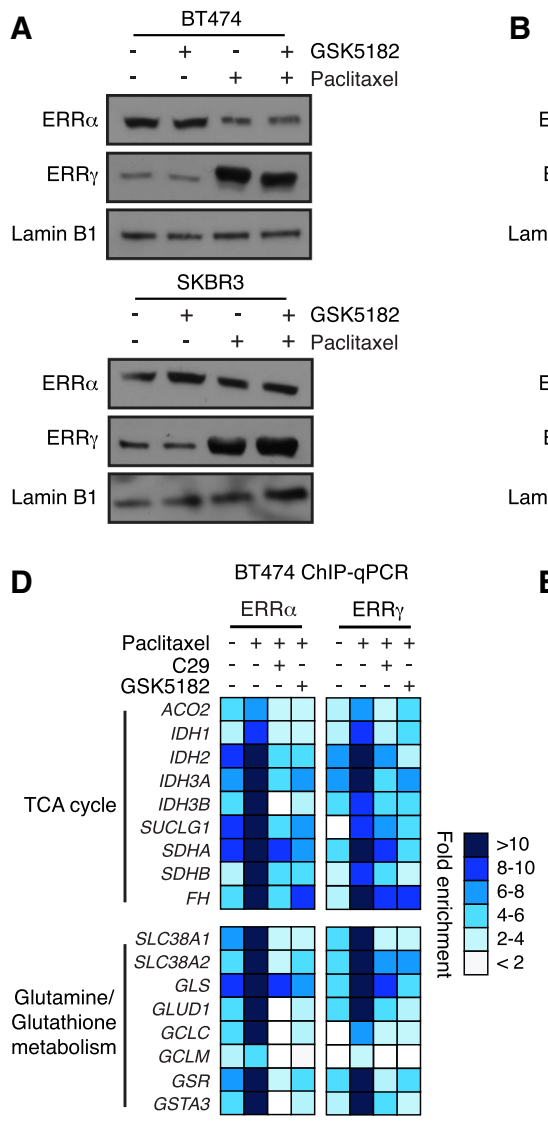

$\mathbf{F}$

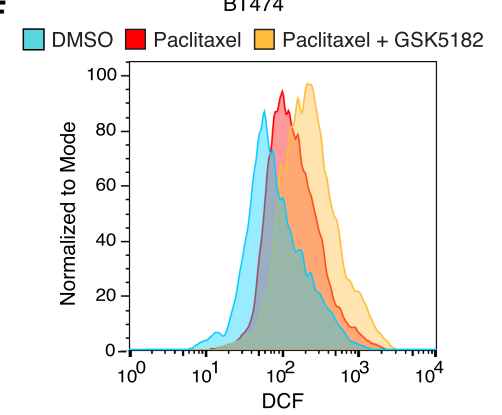

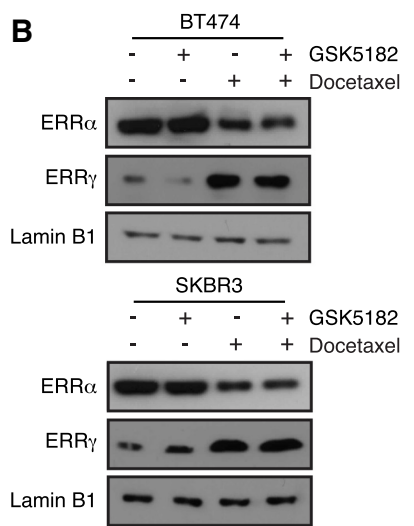

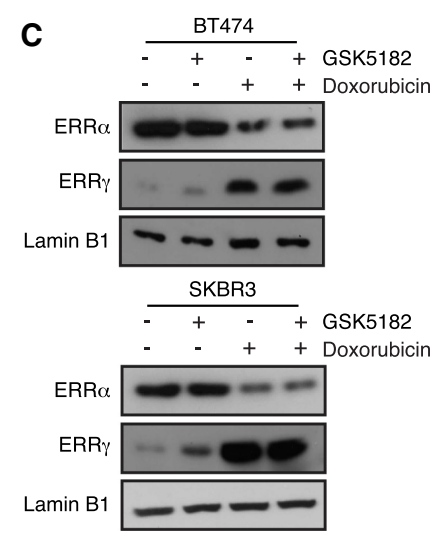

E
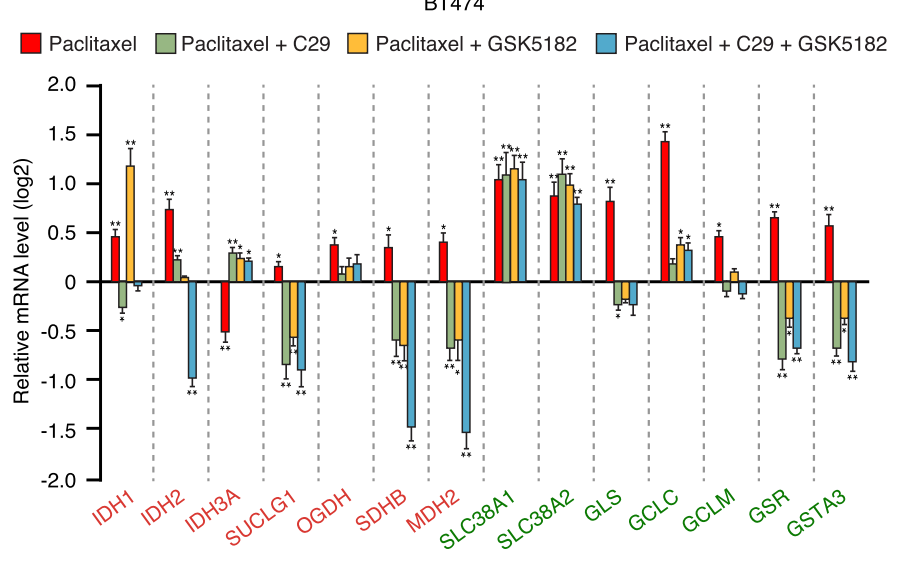

G

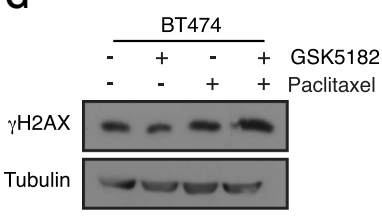

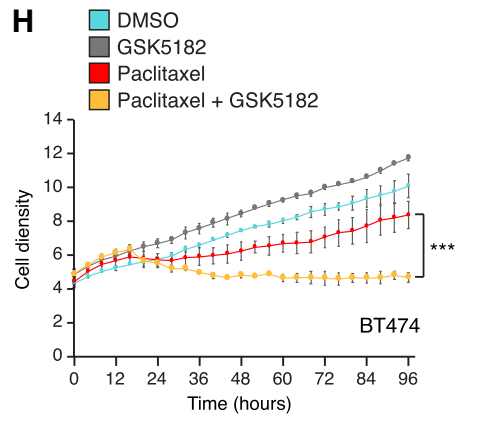

Figure 6. Inhibition of ERR $\gamma$ increases paclitaxel-induced cytotoxicity. $(A-C)$ Immunoblots showing ERR $\alpha$ and ERR $\gamma$ protein levels following treatment of BT474 (top) and SKBR3 (bottom) cells with the chemotherapeutic drugs paclitaxel $(A)$, docetaxel $(B)$, or doxorubicin $(C)$ in combination with the selective ERR $\gamma$ inverse agonist GSK5182. (D) Standard ChIP-qPCR analyses in BT474 cells showing the effect of a 1-h paclitaxel treatment on ERR $a$ and ERR $\gamma$ binding to TCA cycle and glutamine/glutathione metabolism cotarget genes with or without pretreatment with C29 or GSK5182. (E) qRT-PCR analysis of a subset of ERRa/ $\gamma$ TCA cycle and glutamine/glutathione target genes following treatment with paclitaxel \pm C29 or GSK5182 in BT474 cells. $(F, G)$ Levels ROS detected by fluorescence of oxidized 2', $7^{\prime}$-dichlorofluorescein $(\mathrm{DCF})(F)$ and the DNA damage marker $\gamma \mathrm{H} 2 \mathrm{AX}(G)$ in BT474 cells following treatment with paclitaxel and/or the ERR $\gamma$ inhibitor GSK5182. (H) Pharmacological inhibition of ERR $\gamma$ with GSK5182 sensitizes BT474 cells to paclitaxel-induced cell death. Data represent mean \pm SEM. $\left(^{*}\right) P<0.05 ;\left(^{* *}\right) P<0.01 ;\left({ }^{* * *}\right) P<0.001$, Student's $t$-test. 
stress. While rotenone largely favored the induction of ERR $\gamma$ recruitment (Fig. 5C), paclitaxel enhanced ERRa and ERR $\gamma$ DNA binding to a similar extent (Fig. 6D). Nonetheless, both rotenone- and paclitaxel-induced ERR $\gamma$ binding was dependent on ERRa, as the increase in ERR $\gamma$ occupancy in response to drug-induced oxidative stress was strongly prevented by $\mathrm{C} 29$ treatment (Figs. 5C and $6 \mathrm{D})$. Consistent with the effects observed with rotenone, treatment of cells with either C29 or GSK5182 was sufficient to block or reverse paclitaxel-induced upregulation of several TCA cycle and glutamine/glutathione pathway genes (Fig. 6E). Remarkably, inhibiting ERR $\gamma$ with GSK5182 further increased paclitaxel-induced ROS and $\gamma \mathrm{H} 2 \mathrm{AX}$ levels concomitant with aggravation of chemotherapy-induced cytotoxicity (Fig. 6F-H; Supplemental Fig. S6B-E). In addition, we found that either ERR $\alpha$ inhibition by C29 or ERR $\alpha$ overexpression could augment paclitaxel-induced cytotoxicity (Supplemental Fig. S6F,G). The former observation can be attributed to the loss of ERR $\gamma$-stimulated transcriptional activity by C29 and the latter possibly due to intensified mitochondrial activity. Together, the data support the benefit of perturbing ERR isoform activity to hamper the up-regulation of ROS mitigation strategies and thus improve response to chemotherapy.

\section{An ERR $\alpha / \gamma$ targeted ROS homeostasis gene signature correlates with poor chemotherapeutic response of $B C a$ patients}

Cancer cells have rewired metabolism and redox balance to fuel growth and proliferation (Cairns et al. 2011). To gain insight into some of the metabolic programs altered in tumors prior to treatment, we extracted available transcriptome data of $\mathrm{BCa}$ patients prior to chemotherapy and/or radiotherapy, both inducers of oxidative stress, from the large Molecular Taxonomy of Breast Cancer International Consortium (METABRIC) cohort (Pereira et al. 2016). We classified the samples into two categories consisting of patients with either bad or good treatment response as determined by having either a low overall survival $(<24 \mathrm{mo}, \mathrm{n}=133$ ) or high overall survival (>100 mo, $\mathrm{n}=1006$ ), respectively. Gene set enrichment analysis (GSEA) showed significant up-regulation of several metabolic KEGG pathways including glycolysis, 1-carbon metabolism, and pyrimidine metabolism associated with the pentose phosphate pathway in tumors of patients that had a bad overall survival (Fig. 7A). This is consistent with more aggressive tumors having higher metabolic activities. In the same cohort, we also found a significant positive association of ERRa mRNA levels (ESRRA) in patients with low overall survival (Fig. 7B). Based on previous work, up-regulating ERRa activity stimulates metabolic flexibility and ability to use diverse substrates as energetic fuels, thus facilitating tumorigenesis (Deblois et al. 2009, 2016; Chang et al. 2011; Audet-Walsh et al. 2016; Park et al. 2016, 2019). We next analyzed a second cohort of BCa patients with available tumor RNA expression profiles after chemotherapy and their associated response to standard anthracycline and taxane-based chemotherapy
(GSE28844) (Vera-Ramirez et al. 2013). Despite the small cohort, GSEA determined an up-regulation of glutathione metabolism in patients who did not respond well to treatment (Fig. 7C), emphasizing the importance of cancer cells to enhance antioxidant production and defense response to resist chemotherapy-induced cytotoxicity. Concordantly, our ERRa/ $\gamma$ targeted ROS homeostasis gene set (118 genes), extended to include the validated cotarget genes $F H$, SDHA, SDHB, and GCLM (Fig. 3; Supplemental Table S3), was also found significantly up-regulated by GSEA in postchemotherapy BCa tumor samples of poor responders (Fig. 7C). Within the GSEA core enrichment genes contributing the greatest to the up-regulation were genes linked to the TCA cycle $(I D H 2, F H$, and $O G D H L)$, glutathione transport/metabolism (SLC38A1, GSR, GSTA3, and GGT6) as well as the methionine cycle (MAT1A and AHCY), the latter which can supply cysteine required for glutathione synthesis. The data indicate that tumors more apt at maintaining ROS homeostasis in response to ROS-based chemotherapy, a program controlled by modulation of ERR $\alpha$ and ERR $\gamma$ transcriptional activity, are less likely to respond to treatment.

Finally, given the critical importance of our work implicating ERR $\gamma$ up-regulation as an ROS defense response, we sought to test the efficacy of combining the ERR $\gamma$ inhibitor GSK5182 with paclitaxel in a preclinical setting. Patient-derived organoids are emerging as a valuable primary tumor drug response tool as they closely represent a patient's disease phenotypes (Weeber et al. 2017; Roelofs et al. 2019). As such, we established PDX organoids (PDXOs) from two women with triple negative $\mathrm{BCa}$ which grow well in $3 \mathrm{D}$ cultures given the aggressive nature of this molecular subtype. We first confirmed that paclitaxel induces ERR $\gamma$ levels while reducing ERR $\alpha$ expression in the triple-negative BCa cell line MDA-MB231 (Fig. 7D), recapitulating the effects of paclitaxel observed in the HER2 ${ }^{+}$(SKBR3) and Luminal B (BT474) cell lines. Importantly, while GSK5182 alone had no significant effect, the increased cytotoxicity of PDX1886 and PDX2215 organoid models to increasing doses of paclitaxel was further exacerbated by cotreatment with the ERR $\gamma$ inhibitor GSK5182 (Fig. 7E,F). Collectively, the data support ERR $\gamma$ as an actionable drug target for combinatorial ROS-based therapy in $\mathrm{BCa}$ patients.

\section{Discussion}

To achieve ROS homeostasis and avoid oxidative stress, a balance between ROS production and its scavenging is required. In this work, we demonstrate that the nuclear receptors ERRa and ERR $\gamma$ are redox sensors that play essential roles in the control of ROS-related metabolism. ChIP-seq analysis, transcriptional profiling, and metabolite pathway tracing using SIRM were used to delineate the underlying mechanisms involved in ROS regulation by the two ERR isoforms. In the basal state, inhibiting ERRa diminishes total cellular ROS levels, an effect attributed to the metabolic rewiring and shift of glutamine flux away from TCA cycle entry and toward its 
A

\begin{tabular}{llcc} 
Low vs High Overall Survival & & & \\
& & & \\
GSEA: UPREGULATED METABOLIC KEGG PATHWAYS & NES & p-value & q-value \\
\hline GLYCOSPHINGOLIPID_BIOSYNTHESIS & 1.855 & 0.000 & 0.020 \\
GALACTOSE_METABOLISM & 1.669 & 0.004 & 0.132 \\
GLYCOLYSIS_GLUCONEOGENESIS & 1.528 & 0.014 & 0.250 \\
ONE_CARBON_POOL_BY_FOLATE & 1.518 & 0.019 & 0.193 \\
PYRIMIDINEMETABOLISM & 1.502 & 0.025 & 0.194 \\
GLYOXYLATE_AND_DICARBOXYLATE_METABOLISM & 1.477 & 0.048 & 0.231 \\
\hline
\end{tabular}

B

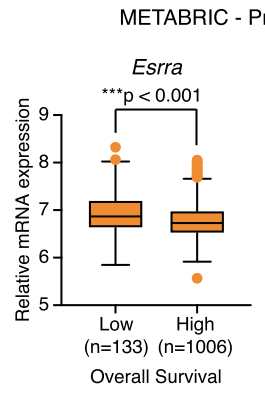

D

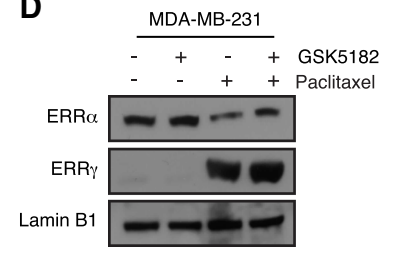

E
C

Bad vs Good response to anthracycline+taxane-based chemotherapy GSEA: UPREGULATED METABOLIC KEGG PATHWAYS NES p-value q-value $\begin{array}{llll}\text { PYRIMIDINE_METABOLISM } & 1.656 & 0.006 & 0.036 \\ \text { GLUTATHIONE_METABOLISM } & 1.462 & 0.040 & 0.146\end{array}$

GSEA: UPREGULATED ERR ROS-RELATED GENE SET NES p-value $q$-value ERR $\alpha /$ /ERRY-TARGETED ROS HOMEOSTASIS GENE SET (122) $1.417 \quad 0.020 \quad 0.020$ ERR $\alpha$ /ERR $y$-TARGETED METABOLIC GENE SET Post-chemo tumors Post-chemo tumors

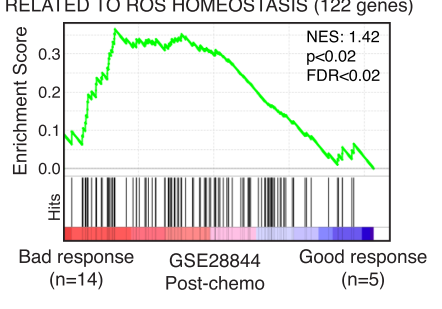
Bad Good

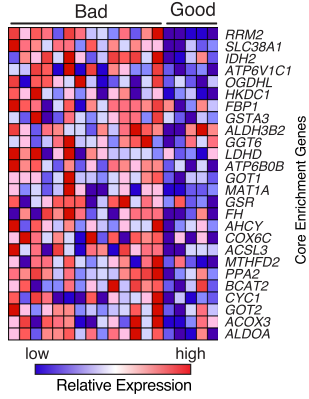

PDX1886 Organoids

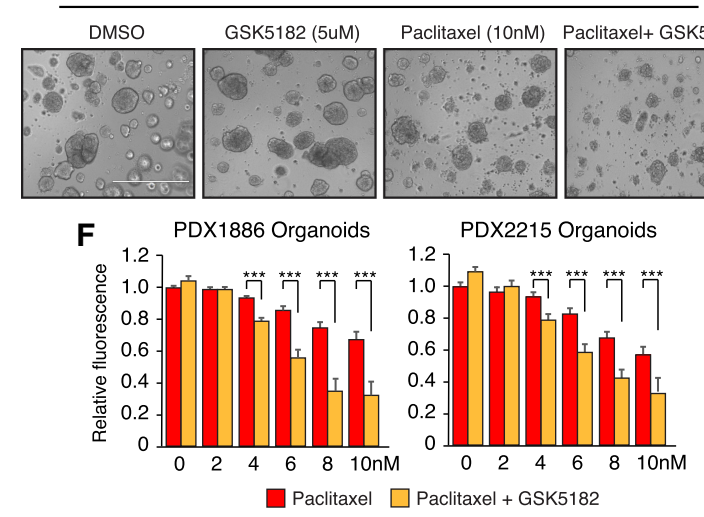

Figure 7. Pharmacological inhibition of ERR $\gamma$ by GSK5182 increases response of PDXOs to chemotherapy. (A) GSEA-determined metabolic KEGG pathways up-regulated in tumors taken prechemotherapy from BCa patients who displayed a low overall survival $(<24 \mathrm{mo})$ compared with those with a high overall survival (>100 mo) taken from the METABRIC cohort. $(B)$ Box and whiskers plot (Tukey style) of mRNA expression levels of ERR $\alpha(E S R R A)$ and ERR $\gamma(E S R R G)$ in tumor samples from $A$. (C, top panel) Significant up-regulated KEGG metabolic pathways by GSEA in postchemotherapy tumors of BCa patients that exhibited a negative (bad) response to an anthracycline/taxane-based therapy compared with those that had a favorable (good) response (GSE28844). (Bottom panel) GSEA established a significant up-regulation of a 122-gene ERR $\alpha / \gamma$ targeted signature identified in Figure 3 related to ROS homeostasis in postchemotherapy tumor samples from BCa patients who exhibited the worst response to treatment (GSE28844). (D) ERR $a$ and ERR $\gamma$ protein levels in the triple-negative BCa cell line MDA-MD-231 following treatment with paclitaxel and/or the ERR $\gamma$ inhibitor GSK5182. (E) Representative images showing a greater reduction in triple-negative PDXO densities with a paclitaxel $(10 \mathrm{nM})+\mathrm{GSK} 5182(5 \mu \mathrm{M})$ regimen compared with paclitaxel alone. $(F)$ Relative cell density measurements of PDXO cultures from two triple-negative BCa patients treated with GSK5182 $(5 \mu \mathrm{M})$ and increasing doses of paclitaxel. Data represent mean \pm SEM. $\left(^{* * *}\right) P<0.001$, Student's $t$-test.

consumption for glutathione production. Notably, we demonstrate that targeting ERR $\gamma$ can block this protective mechanism against ROS detoxification mediated by loss of ERR $a$ function as ERR $\alpha$ and ERR $\gamma$ were found to inversely regulate the flux of glutamine towards reductive carboxylation and glutathione biosynthesis. In contrast to the identified opposing roles of ERR $a$ and ERR $\gamma$ observed under normal conditions, oxidative stress-induced cells were found to require activation of both receptors to set in motion an adaptive antioxidant stress response. Pharmacological inhibition of ERR $\gamma$ by GSK5182 or ERRa by C29 were both found to block the induction of ERR $\gamma$ target gene occupancy in response to the ROS-pro- voking mitochondrial disruptor rotenone and the chemotherapeutic drug paclitaxel. As such, inhibiting ERRa or ERR $\gamma$ impaired or reversed the stimulatory action of rotenone or paclitaxel on several TCA cycle and glutamine/ glutathione pathway genes and consequently aggravated the oxidative stress-induced cytotoxic effects, thus sensitizing cells to ROS-promoting drugs. The benefits of targeting ERR $\gamma$ up-regulation mediated by ERRa loss of function under normal conditions or mediated by oxidative stress induced by paclitaxel or rotenone treatment are summarized in Figure 8.

It is well known that most chemotherapeutics induce cytotoxic effects on cancer cells via the induction of 
A

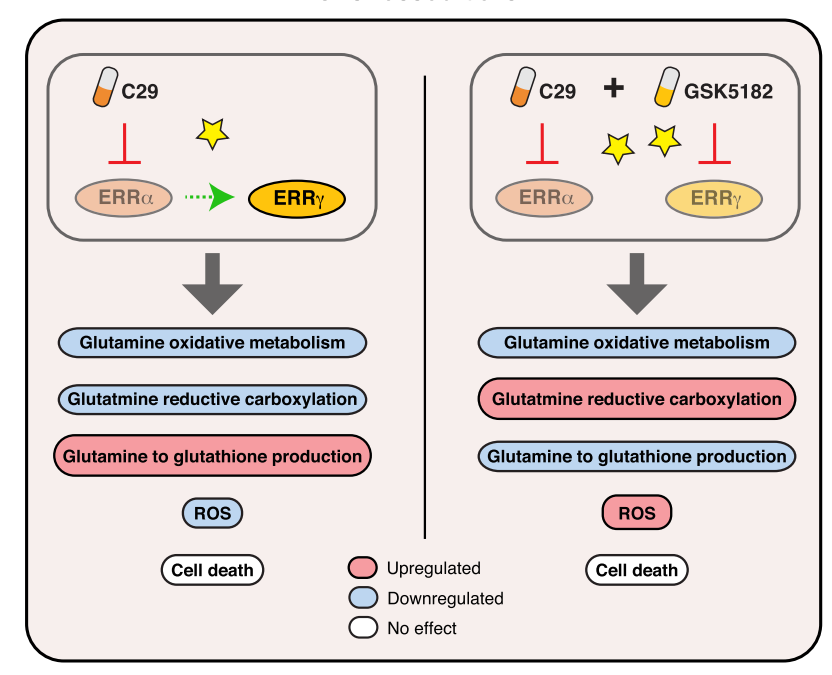

B

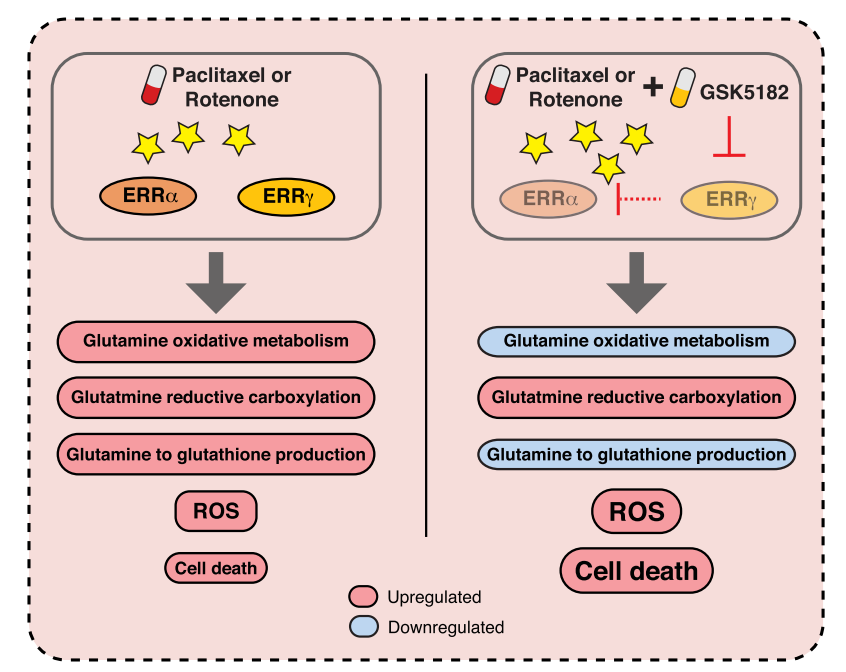

Figure 8. ERR $\gamma$ is a targetable ROS defense mechanism. (A, left) ERRa loss of function by C29 promotes ERR $\gamma$ activity leading to an induction of glutamine-driven glutathione production and suppression of ROS. (Right) Concomitant pharmacological inhibition of ERRa by C29 and ERR $\gamma$ by GSK5182 reverses those effects with no consequence on cell death. (B, left) Induction of oxidative stress by the chemotherapeutic drug paclitaxel or the mitochondrial disruptor rotenone induces cell death. As a ROS defense mechanism, cells respond by increasing the activity of both ERR $\alpha$ and ERR $\gamma$ to rewire glutamine fate and enhance glutathione production. (Right) Inhibiting ERR $\gamma$ activity with GSK5182 abrogates this adaptive ERR-driven response, thus sensitizing cells to the cytotoxic effects of chemotherapeutic agents. Loss of ERR $\alpha$ or ERR $\gamma$ activity is represented by decreased opacity of the proteins. ROS levels are represented by stars. Solid lines represent direct effects of the drugs on each ERR isoform while dashed lines indicate indirect regulation of the activity of one ERR isoform by another.

oxidative stress and cellular damage. However, cancer cells have evolved mechanisms to re-establish redox balance fueling drug resistance and survival. By analyzing a large cohort of BCa patients, we observed that tumors with a high metabolic activity are more resistant to chemotherapy and this correlates with high levels of ERRa expression. Moreover, analysis of an independent cohort taken after chemotherapy revealed an up-regulation of glutathione metabolism, reinforcing antioxidant production as an important ROS defense mechanism. Likewise, a 122-gene ERRa $/ \gamma$ targeted metabolic signature related to ROS homeostasis including the glutamine/glutathione pathway was also strongly up-regulated in BCa tumors after chemotherapy. These data are thus in accordance with the notion of metabolic plasticity, which allows cancer cells to rapidly adapt their metabolism to external stimuli such as chemotherapeutic agents (Jia et al. 2018).

Identifying the molecular effectors of ROS defense mechanisms may be exploited as targeted therapies for cancer and other diseases. Known transcription factors previously shown to favor ROS scavenging in cancer cells and tumor progression include most notably nuclear factor erythroid 2-related factor 2 (NRF2) and hypoxia-inducible factor 1- $\alpha$ (HIFl $\alpha$ ) (Trachootham et al. 2006; Gao et al. 2007; DeNicola et al. 2011; Ren et al. 2011). Here, we identified ERR $\gamma$ as a druggable cancer metabolic vulnerability to oxidative stress. Notably, combining the selective ERR $\gamma$ inhibitor GSK5182 with the chemotherapeutic drugs paclitaxel, docetaxel, and doxorubicin, all of which up-regulated ERR $\gamma$ expression and activity accentuating the chemotherapy-induced cytotoxic effects in BCa cells.
Accordingly, cotreatment of GSK5182 with paclitaxel was found significantly more effective at reducing the proliferation of two aggressive BCa PDXO models than paclitaxel alone, supporting the potential use of ERR $\gamma$ inhibitors in clinical settings.

In conclusion, our study uncovered that ERRa and ERR $\gamma$ function as ROS sensors and are codependent on each other's activity to induce an oxidative stress response. We demonstrated that ERR $\gamma$ increased expression and activity may serve as a hallmark of oxidative stress and can be exploited therapeutically as a metabolic vulnerability to sensitize BCa cells to chemotherapy. Given the association of oxidative stress in a wide range of diseases including other cancer types, neurological pathologies and aging, it will therefore be of great interest to decipher the precise molecular mechanisms by which fluctuating ROS levels influence ERR activity.

\section{Materials and methods}

Cell lines and reagents

BT474, SKBR3, and MDA-MB-231 cell lines were originally obtained from American Type Culture Collection (ATCC) and maintained in DMEM supplemented with $10 \%$ fetal bovine serum (FBS). ERRa WT and null (KO) MEFs were maintained in DMEM supplemented with $10 \%$ fetal bovine serum (FBS). GSK5182, rotenone, TTFA [4,4,4-trifluoro-1-(2-thienyl)-1,3-butanedione], oligomycin, MitoQ and MG132 were purchased from Sigma-Aldrich. Pools of siRNAs against ERR $\alpha, E R R ~ \gamma$, and control (siC) (ON-Target-Plus siRNA pool) were obtained from Dharmacon. Transfections were performed with Hiperfect (Qiagen). 
SKBR3 cells overexpressing ERR $\alpha$ were generated by infecting cells with the pLX3.17-ERRa lentiviral plasmid for Sigma. ERR $\alpha$ and ERR $\gamma$ ChIP assays were performed using an antiERRa rabbit monoclonal antibody (Abcam 2131-1), anti-ERR $\gamma$ rabbit antibody (from Dr A. Kralli) and control antirabbit IgG antibody (Santa Cruz Biotechnology sc-2027). The ERR $\gamma$ ChIP sequencing was performed with an anti-ERR $\gamma$ rabbit antibody from Dr R. Evans (Salk Institute). Western blots on cell extracts were performed using the following antibodies: ERR $\alpha$ (1:500; Abcam 2131-1), ERR $\gamma$ (1:10,000; from Dr A. Kralli), $\gamma \mathrm{H} 2 \mathrm{AX}$ (1:1000), Tubulin (1:10000), and Lamin B1 (1:1000).

\section{Chromatin immunoprecipitation (ChIP) and ChIP sequencing}

For ChIP analyses after treatment with C29 or GSK5182, chromatin was prepared from BT474 cells cultured for $24 \mathrm{~h}$ with $5 \mu \mathrm{M}$ drugs as indicated. For ChIP analysis with rotenone or paclitaxel, cells were treated for $1 \mathrm{~h}$ with $1 \mu \mathrm{M}$ of drug. The ChIP primers are listed in Supplemental Table S4. Standard ChIP was performed as described previously (Audet-Walsh et al. 2016). Quantification of ChIP enrichment by real-time quantitative PCR was carried out using the LightCycler480 instrument (Roche). ChIP binding fold enrichments were normalized against the background enrichment from anti-IgG antibody ChIPs as well as the enrichment obtained from two negative control regions (unbound regions). Representative heat map of three independent experiments performed in triplicates are shown. Statistical significance of standard ChIP is obtained with unpaired Student's $t$-tests.

For duplicate human ERR $\gamma$ ChIP-seq experiments, following ERR $\gamma$ ChIP with Dynabeads (Life Technologies) and purification of DNA with a purification kit (Qiagen), libraries of enriched DNA segments were prepared per the ChIP-seq library protocol by Illumina as described previously (Audet-Walsh et al. 2016) and subsequently sequenced at the McGill University and Génome Québec Innovation Centre. Input DNA was used as a control ChIP-seq. Sequences were aligned to the human genome database $(\mathrm{Hg} 19)$ using BWA version 0.5.9. Peaks were called using MACS software version 1.4.1. with default parameters using sequenced libraries of input DNA as control. Peak annotation, tag directory, and bed file generation was performed with the HOMER package version 3.18. The annotated peaks identified are available in the Supplemental Table S2.

\section{Cell proliferation assay}

For proliferation assays, $2 \times 10^{3}$ cells were seeded in 96-well plates. The next day, cells were treated with C29 (5 $\mu \mathrm{M})$, GSK5182 $(5 \mu \mathrm{M})$, or vehicle in combination with $2 \mathrm{nM}$ paclitaxel, $6 \mathrm{nM}$ doxorubicin, or $2 \mathrm{nM}$ docetaxel and transferred to an IncuCyte ZOOM Live cell analysis system (Essen BioScience). Proliferation was monitored using IncuCyte ZOOM phase-contrast quantification software (2014B). For proliferation assay with ERRa overexpression, cells were infected with the pLX3.17ERRa plasmid and selected for $4 \mathrm{~d}$ before being seeded, treated with $2 \mathrm{nM}$ paclitaxel, and monitored as previously described.

\section{ROS quantification}

Cells $\left(1 \times 10^{5}\right)$ were seeded in six-well plates. Treatment with $5 \mu \mathrm{M}$ C2 29 or $5 \mu \mathrm{M}$ GSK5182 were carried out for $24 \mathrm{~h}$. Treatment with $10 \mu \mathrm{M}$ rotenone or $10 \mu \mathrm{M}$ paclitaxel was performed for $4 \mathrm{~h}$. Cells were then stained with $5 \mu \mathrm{M} \mathrm{H}_{2}$ DCFDA (Invitrogen) for $30 \mathrm{~min}$ at $37^{\circ} \mathrm{C}$. The cells were washed with phosphate buffer saline (PBS) and subjected to a flow cytometric analysis at ex- citation wavelength $492 \mathrm{~nm}$ and emission of $520 \mathrm{~nm}$ using BD FacsCalibur.

\section{Quantitative RT-PCR}

RNA was extracted and purified using the RNeasy minikit (Qiagen) or the Aurum Total RNA minikit (Bio-Rad) following the manufacturers' protocols. Reverse transcriptase reactions were performed using SuperScript II (Invitrogen) or iScript cDNA Synthesis Kit (Bio-Rad). Samples were then analyzed by qRT-PCR with SYBR Green-based qRT-PCR on a LightCycler 480 instrument (Roche). Relative mRNA expression values were normalized to the average expression of TBP and PUM1. The qRT-PCR primers are listed in Supplemental Table S4.

\section{GC/MS, LC/MS, and mass isotopomer distribution analysis}

Steady-state abundances of GSH and GSSG were determined using LC/MS systems (unlabeled media). Seventy percent to $80 \%$ confluent $10-\mathrm{cm}$ plates of cultured cells were washed with 150 $\mathrm{mM}$ ammonium formate at $4^{\circ} \mathrm{C}$ and quenched in 50\% HPLCgrade methanol at $-20^{\circ} \mathrm{C}$ on dry ice, after which the cell slurry was quickly transferred to tubes equilibrated in liquid nitrogen. Then, these were phase-separated using acetonitrile, water and dichloromethane after vigorous bead beating and vortexing. The aqueous phase was collected and dried in a cold trap at $-1^{\circ} \mathrm{C}$. Once dry, pellets were maintained at $-80^{\circ} \mathrm{C}$ and solubilized in HPLC water immediately before injection into an Agilent 6430 Triple Quadrupole LC/MS system coupled to ultra-high-pressure liquid chromatography (UHPLC; 1290 Infinity LC system) separation for fast targeted analysis. Data were analyzed using Masshunter software.

For SIRM, cells were seeded in six-well dishes to achieve $70 \%-$ $80 \%$ cell confluency after $48 \mathrm{~h}$. Twenty-four hours after seeding, cells were treated with $5 \mu$ M C29 or GSK5182 for another $24 \mathrm{~h}$. Then, the medium was replaced by DMEM without glutamine supplemented with $10 \%$ FBS, $1 \times$ sodium pyruvate and $4 \mathrm{mM}$ $\left[\mathrm{U}-{ }^{13} \mathrm{C}\right]$-glutamine. A 2 -h pulse of labeled glutamine was used for TCA cycle flux analysis and a 6-h pulse was used for glutathione flux measurements. C29 and GSK5182 were present in the media throughout. Metabolites were then purified as for steadystate metabolites as described above.

\section{Determination of extracellular metabolite concentration}

For determination of extracellular concentrations of glutamine and glutamate, $1 \times 10^{5}$ cells were seeded in six-well plates and treated with $5 \mu \mathrm{M}$ C29 or GSK5182 for $72 \mathrm{~h}$ before collecting the supernatants. Metabolite concentrations were assessed with the Bioprofile 400 analyzer according to the manufacturer's protocol.

\section{Immunofluorescence of 8-oxoguanine}

Cells were plated on coverslips and treated with $5 \mu \mathrm{M}$ C29 or GSK5182 for $24 \mathrm{~h}$ prior to fixation with $4 \%$ paraformaldehyde in PBS for $10 \mathrm{~min}$ at $4^{\circ} \mathrm{C}$. After washing with PBS, cells were treated with RNase A (100 ug/mL; Sigma-Aldrich 109169001) in $10 \mathrm{mM}$ Tris- $\mathrm{HCl}(\mathrm{pH} 7.5)$ and $15 \mathrm{mM} \mathrm{NaCl}$ for $1 \mathrm{~h}$ at $37^{\circ} \mathrm{C}$. Cells were then permeabilized with PBS $/ 0.1 \%$ Triton X-100 for $10 \mathrm{~min}$, rinsed, and treated with $2 \mathrm{M}$ HCL for $10 \mathrm{~min}$ for DNA denaturation. Cells were then washed with $50 \mathrm{mM}$ Tris-base for $10 \mathrm{~min}$ at room temperature. For antibody staining, cells were washed with $3 \%$ bovine serum albumin (BSA) in PBS (PBS/BSA) and incubated overnight with an antibody against 8-oxoguanine (mouse; 
1/100; Abcam ab62623). After primary antibodies, cells were washed three times in PBS/BSA and incubated with the secondary antibody (1/2000; AlexaFluor 488 goat antimouse; Molecular Probes-Invitrogen) for $1 \mathrm{~h}$ at room temperature. Finally, cells were rinsed twice with PBS alone and once with PBS containing 300 $\mu M$ DAPI for $10 \mathrm{~min}$. Images were captured with a Zeiss LSM710 confocal laser microscope and were processed and quantified with ImageJ.

\section{Cell viability test}

Cells $\left(1 \times 10^{5}\right)$ were seeded in six-well plates $24 \mathrm{~h}$ prior treatment with $5 \mu \mathrm{M}$ C29 or GSK5182 in combination with rotenone at the indicated concentrations for $36 \mathrm{~h}$. Supernatant containing dead cells was collected and surviving cells were trypsinized. After centrifugation, cells were rinsed, stained with trypan blue and counted with the Bio-Rad TC20 cell counter to evaluate percentage of dead cells over total cell count.

\section{Clinical data set analyses}

Biostatistical analyses were conducted on the previously published METABRIC data set from which we isolated gene expression data (before treatment) of breast cancer patients who received neoadjuvant chemotherapy or radiotherapy (Pereira et al. 2016). Patients were then separated in two groups based on their overall survival (OS) with a group exhibiting high OS reflecting good response to treatment $(>100 \mathrm{mo}, n=1006)$ and a group with low OS considered as a bad response to treatment $(<24 \mathrm{mo}, \mathrm{n}=133)$. Pathway analysis was performed with the GSEA software tool (http://www.broadinstitute.org/gsea/index .jsp) by comparing gene expression profiles of each group to identify significantly enriched metabolic KEGG pathways within the Molecular Signature Database (MSigDB, v6.2). Comparisons of ERR $\alpha$ (ESRRA) and ERR $\gamma(E S R R G)$ mRNA levels between the two groups were performed with Prism 8 software.

A second cohort of patients (GSE28844) was then used to identify the KEGG metabolic pathways enriched after chemotherapy in patients that displayed a bad response (GSE28826), according to Miller and Payne grade, versus those that exhibited a good response (GSE28694) to a standard anthracycline and taxane-based treatment regimen (Vera-Ramirez et al. 2013). Pathway analysis was performed with GSEA by comparing good versus bad response gene expression profiles after chemotherapy.

To design the ERR $/ \gamma$ targeted metabolic gene set, we first isolated the genes identified as cobound by ERR a and ERR $\gamma^{+/-} 20 \mathrm{~kb}$ from the transcription start site of genes from the ChIP sequencing data sets on one side and included four genes (FH, SDHA, $S D H B$, and GCLM) missed in the ERR $\gamma$ ChIP-seq analysis but validated as bona fide targets by ChIP-qPCR. Then, we compiled a list of genes from metabolic KEGG pathways associated with ROS homeostasis as indicated in Figure 3A and listed in Supplemental Table S1, including 14 glutamine transporters. This curated gene set was compared with the list of ERR $\alpha / \gamma$ cotarget genes resulting in an ERR targeted signature of 122 genes implicated in ROS homeostasis (Supplemental Table S3).

\section{PDX-derived organoids}

Tumor specimens were obtained from two women diagnosed with triple-negative $\mathrm{BCa}$ and patient-derived xenografts (PDXs) were generated. Tissues were collected with informed consent under REB-approved protocols at the McGill University Health Centre. Mice were maintained and treated in accordance with the Facility Animal Care Committee at the Goodman Cancer Re- search Centre of McGill University (2014-7514). When the PDX tumors reached $10 \mathrm{~mm}$ in the largest dimension, tumors were harvested for the establishment of PDX-derived organoids (PDXOs). Briefly, PDX tumors were dissociated into single cells suspensions using a tumor dissociation kit (Miltenyi Biotec) in combination with the gentleMACS Dissociator (Miltenyi Biotec). Cell pellets were resuspended in $1 \mathrm{~mL}$ of organoid media (Sachs et al. 2018) and seeded in 12-well Matrigel (Corning) coated plates. Organoid media was refreshed every $3 \mathrm{~d}$ until organoid formation.

For PDXO drug treatments, $30 \mu \mathrm{L}$ of Matrigel were seeded in a CELLSTAR u-clear white wall 96-well plates (Greiner Bio-One) and incubated for $30 \mathrm{~min}$ at $37^{\circ} \mathrm{C}$ and $5 \% \mathrm{CO}_{2}$. PDXOs were dissociated into single cells and the Matrigel was then overlaid with $100 \mu \mathrm{L}$ of organoid medium containing 5\% Matrigel (8000 cells/ well). Four days after seeding, the medium was removed and replaced by $100 \mu \mathrm{L}$ of drug-containing organoid media. At the end of the treatment $(4 \mathrm{~d})$, the medium was removed and replaced with $100 \mu \mathrm{L}$ of complete human organoid media containing $10 \%$ CellTiter-Blue (Promega). Plates were placed back in the incubator for an additional $4 \mathrm{~h}$. Viability readings were obtained using the FLUOstar Omega plate reader (BMG Labtech).

Statistical analyses

Statistical analyses were performed with Prism 8 software (GraphPad). Data are expressed as means \pm SEM unless otherwise stated. Differences between two groups were determined by unpaired Student $t$ test (two-tailed).

Accession numbers

ERR $\gamma$ ChIP-seq data have been deposited in the NCBI Gene Expression Omnibus (GEO; https://ncbi.nlm.nih.gov/geo/) under the accession number GSE144224. ERR $\mathrm{ChIP}$-seq data were previously obtained and available under the GEO accession number GSE75876.

\section{Acknowledgments}

We acknowledge contributions from the Metabolomics Core Facility (MCF) of the Goodman Cancer Research Centre, as well as technical assistance from Dr. Daina Avizonis, Mariana De Sa Tavares Russo, Gaëlle Bridon, and Luc Choinière. We thank Dr. Anastasia Kralli and Dr. Ronald M. Evans for kindly providing the custom-made ERR $\gamma$ antibodies. We thank members of the V.G. laboratory for discussions, and Dr. David J. Papadopoli for guidance with metabolic studies. This work was supported by a Terry Fox Research Institute Program Project Team Grant on Oncometabolism (V.G. and J.St-P.), Foundation Grants from the Canadian Institutes of Health Research (CIHR) to V.G. and M.P., and an operating grant from CIHR to J.St-P. (MOP106603). M.V. is recipient of a postdoctoral fellowship from CIHR. S.M. is supported by a Vanier Canada Graduate Scholarship-CIHR. E.A.-W. was a recipient of a postdoctoral fellowship from CIHR.

Author Contributions: M.V. and V.G. conceived the study. M.V., S.M., H.K., M.P., and J.St-P. developed the methodology. M.V., S.M., C.R.D., C.S., H.K., X.L., G.B., and E.A.-W. performed the investigation. M.V., C.R.D., and V.G. wrote the manuscript. M.V. and C.R.D. visualized the study. V.G., M.P., and J.St-P. acquired funding. V.G. supervised the study. 


\section{References}

Audet-Walsh E, Giguère V. 2015. The multiple universes of estrogen-related receptor $a$ and $\gamma$ in metabolic control and related diseases. Acta Pharmacol Sin 36: 51-61. doi:10.1038/aps .2014 .121

Audet-Walsh E, Papadopoli DJ, Gravel SP, Yee T, Bridon G, Caron M, Bourque G, Giguère V, St-Pierre J. 2016. The PGC-1 $\alpha /$ ERR $\alpha$ axis represses one-carbon metabolism and promotes sensitivity to anti-folate therapy in breast cancer. Cell Rep 14: 920931. doi:10.1016/j.celrep.2015.12.086

Bhutia YD, Ganapathy V. 2016. Glutamine transporters in mammalian cells and their functions in physiology and cancer. Biochim Biophys Acta 1863: 2531-2539. doi:10.1016/j.bbamcr .2015.12.017

Cadet J, Wagner JR. 2013. DNA base damage by reactive oxygen species, oxidizing agents, and UV radiation. Cold Spring Harb Perspect Biol 5: a012559. doi:10.1101/cshperspect a012559

Cairns RA, Harris IS, Mak TW. 2011. Regulation of cancer cell metabolism. Nat Rev Cancer 11: 85-95. doi:10.1038/nrc2981

Chang CY, Kazmin D, Jasper JS, Kunder R, Zuercher WJ, McDonnell DP. 2011. The metabolic regulator ERRa, a downstream target of HER2/IGF-1R, as a therapeutic target in breast cancer. Cancer Cell 20: 500-510. doi:10.1016/j.ccr.2011.08.023

Chao EY, Collins JL, Gaillard S, Miller AB, Wang L, Orband-Miller LA, Nolte RT, McDonnell DP, Willson TM, Zuercher WJ. 2006. Structure-guided synthesis of tamoxifen analogs with improved selectivity for the orphan ERR $\gamma$. Bioorg Med Chem Lett 16: 821-824. doi:10.1016/j.bmcl.2005.11.030

Charest-Marcotte A, Dufour CR, Wilson BJ, Tremblay AM, Eichner LJ, Arlow DH, Mootha VK, Giguère V. 2010. The homeobox protein Prox1 is a negative modulator of ERR $\alpha / P G C-1 \alpha$ bioenergetic functions. Genes Dev 24: 537-542. doi:10.1101/ gad. 1871610

Chaveroux C, Eichner LJ, Dufour CR, Shatnawi A, Khoutorsky A, Bourque G, Sonenberg N, Giguère V. 2013. Molecular and genetic crosstalks between mTOR and ERR $\alpha$ are key determinants of rapamycin-induced non-alcoholic fatty liver. Cell Metab 17: 586-598. doi:10.1016/j.cmet.2013.03.003

Deblois G, Giguère V. 2013. Oestrogen-related receptors in breast cancer: control of cellular metabolism and beyond. Nat ReV Cancer 13: 27-36. doi:10.1038/nrc3396

Deblois G, Hall JA, Perry MC, Laganière J, Ghahremani M, Park M, Hallett M, Giguère V. 2009. Genome-wide identification of direct target genes implicates estrogen-related receptor $\alpha$ as a determinant of breast cancer heterogeneity. Cancer Res 69: 6149-6157. doi:10.1158/0008-5472.CAN-09-1251

Deblois G, Chahrour G, Perry MC, Sylvain-Drolet G, Muller WJ, Giguère V. 2010. Transcriptional control of the ERBB2 amplicon by ERR $\alpha$ and PGC- $1 \beta$ promotes mammary gland tumorigenesis. Cancer Res 70: 10277-10287. doi:10.1158/00085472.CAN-10-2840

Deblois G, Smith HW, Tam IS, Gravel SP, Caron M, Savage P, Labbé DP, Bégin LR, Tremblay ML, Park M, et al. 2016. ERR $a$ mediates metabolic adaptations driving lapatinib resistance in breast cancer. Nat Commun 7: 12156. doi:10.1038/ ncomms 12156

DeNicola GM, Karreth FA, Humpton TJ, Gopinathan A, Wei C, Frese K, Mangal D, Yu KH, Yeo CJ, Calhoun ES, et al. 2011. Oncogene-induced Nrf2 transcription promotes ROS detoxification and tumorigenesis. Nature 475: 106-109. doi:10.1038/ nature10189

Dufour CR, Wilson BJ, Huss JM, Kelly DP, Alaynick WA, Downes M, Evans RM, Blanchette M, Giguère V. 2007. Genome-wide orchestration of cardiac functions by orphan nucler receptors ERR $a$ and $\gamma$. Cell Metab 5: 345-356. doi:10.1016/j.cmet.2007 .03 .007

Eichner LJ, Giguère V. 2011. Estrogen related receptors (ERRs): a new dawn in transcriptional control of mitochondrial gene networks. Mitochondrion 11: 544-552. doi:10.1016/j.mito .2011 .03 .121

Gao P, Zhang H, Dinavahi R, Li F, Xiang Y, Raman V, Bhujwalla ZM, Felsher DW, Cheng L, Pevsner J, et al. 2007. HIF-dependent antitumorigenic effect of antioxidants in vivo. Cancer Cell 12: 230-238. doi:10.1016/j.ccr.2007.08.004

Giguère V. 2008. Transcriptional control of energy homeostasis by the estrogen-related receptors. Endocr Rev 29: 677-696. doi:10.1210/er.2008-0017

Hong E-J, Levasseur M-P, Dufour CR, Perry M-C, Giguère V. 2013. Loss of estrogen-related receptor a promotes hepatocellular carcinogenesis development via metabolic and inflammatory disturbances. Proc Natl Acad Sci 110: 17975-17980. doi:10.1073/pnas.1315319110

Jia D, Park JH, Jung KH, Levine H, Kaipparettu BA. 2018. Elucidating the metabolic plasticity of cancer: mitochondrial reprogramming and hybrid metabolic states. Cells 7: E21. doi:10 $.3390 /$ cells 7030021

Kim JH, Choi YK, Byun JK, Kim MK, Kang YN, Kim SH, Lee S, Jang BK, Park KG. 2016. Estrogen-related receptor $\gamma$ is upregulated in liver cancer and its inhibition suppresses liver cancer cell proliferation via induction of $\mathrm{p} 21$ and p27. Exp Mol Med 48: e213. doi:10.1038/emm.2015.115

Li L, Fath MA, Scarbrough PM, Watson WH, Spitz DR. 2015. Combined inhibition of glycolysis, the pentose cycle, and thioredoxin metabolism selectively increases cytotoxicity and oxidative stress in human breast and prostate cancer. Redox Biol 4: 127-135. doi:10.1016/j.redox.2014.12.001

Misawa A, Inoue S. 2015. Estrogen-related receptors in breast cancer and prostate cancer. Front Endocrinol (Lausanne) 6: 83. doi:10.3389/fendo.2015.00083

Misra J, Kim DK, Choi HS. 2017. ERR $\gamma$ : a junior orphan with a senior role in metabolism. Trends Endocrinol Metab 28: 261272. doi:10.1016/j.tem.2016.12.005

Mootha VK, Handschin C, Arlow D, Xie X, St Pierre J, Sihag S, Yang W, Altshuler D, Puigserver P, Patterson N, et al. 2004. ERR $\alpha$ and GABPA $\alpha / \beta$ specify PGC- $1 \alpha$-dependent oxidative phosphorylation gene expression that is altered in diabetic muscle. Proc Natl Acad Sci 101: 6570-6575. doi:10.1073/ pnas.0401401101

Murray J, Auwerx J, Huss JM. 2013. Impaired myogenesis in estrogen-related receptor $\gamma(E R R \gamma)$-deficient skeletal myocytes due to oxidative stress. FASEB I 27: 135-150. doi:10.1096/fj.12212290

Panieri E, Santoro MM. 2016. ROS homeostasis and metabolism: a dangerous liason in cancer cells. Cell Death Dis 7: e2253. doi:10.1038/cddis.2016.105

Park S, Chang CY, Safi R, Liu X, Baldi R, Jasper JS, Anderson GR, Liu T, Rathmell JC, Dewhirst MW, et al. 2016. ERRa-regulated lactate metabolism contributes to resistance to targeted therapies in breast cancer. Cell Rep 15: 323-335. doi:10 .1016/j.celrep.2016.03.026

Park S, Safi R, Liu X, Baldi R, Liu W, Liu J, Locasale JW, Chang CY, McDonnell DP. 2019. Inhibition of ERRa prevents mitochondrial pyruvate uptake exposing NADPH-generating pathways as targetable vulnerabilities in breast cancer. Cell Rep 27: 3587-3601.e4. doi:10.1016/j.celrep.2019.05.066

Patch RJ, Searle LL, Kim AJ, De D, Zhu X, Askari HB, O'Neill JC, Abad MC, Rentzeperis D, Liu J, et al. 2011. Identification of diaryl ether-based ligands for estrogen-related receptor $a$ as 
potential antidiabetic agents. J Med Chem 54: 788-808. doi:10 $.1021 / \mathrm{jm} 101063 \mathrm{~h}$

Pereira B, Chin SF, Rueda OM, Vollan HK, Provenzano E, Bardwell HA, Pugh M, Jones L, Russell R, Sammut SJ, et al. 2016. The somatic mutation profiles of 2433 breast cancers refine their genomic and transcriptomic landscapes. Nat Commun 7: 11479. doi:10.1038/ncomms11479

Pizzorno J. 2014. Mitochondria-fundamental to life and health. Integr Med (Encinitas) 13: 8-15.

Ray PD, Huang BW, Tsuji Y. 2012. Reactive oxygen species (ROS) homeostasis and redox regulation in cellular signaling. Cell Signal 24: 981-990. doi:10.1016/j.cellsig.2012.01.008

Ren D, Villeneuve NF, Jiang T, Wu T, Lau A, Toppin HA, Zhang DD. 2011. Brusatol enhances the efficacy of chemotherapy by inhibiting the Nrf2-mediated defense mechanism. Proc Nat1 Acad Sci 108: 1433-1438. doi:10.1073/pnas.1014275108

Roelofs C, Hollande F, Redvers R, Anderson RL, Merino D. 2019. Breast tumour organoids: promising models for the genomic and functional characterisation of breast cancer. Biochem Soc Trans 47: 109-117. doi:10.1042/BST20180375

Sachs N, de Ligt J, Kopper O, Gogola E, Bounova G, Weeber F, Balgobind AV, Wind K, Gracanin A, Begthel H, et al. 2018. A living biobank of breast cancer organoids captures disease heterogeneity. Cell 172: 373-386.e10. doi:10.1016/j.cell.2017 .11 .010

Shadel GS, Horvath TL. 2015. Mitochondrial ROS signaling in organismal homeostasis. Cell 163: 560-569. doi:10.1016/j.cell .2015 .10 .001

Sonoda J, Laganière J, Mehl IR, Barish GD, Chong LW, Li X, Scheffler IE, Mock DC, Bataille AR, Robert F, et al. 2007. Nuclear receptor ERR $\alpha$ and coactivator PGC- $1 \beta$ are effectors of IFN- $\gamma$ induced host defense. Genes Dev 21: 1909-1920. doi:10 $.1101 /$ gad.1553007

Trachootham D, Zhou Y, Zhang H, Demizu Y, Chen Z, Pelicano H, Chiao PJ, Achanta G, Arlinghaus RB, Liu J, et al. 2006. Selective killing of oncogenically transformed cells through a ROS-mediated mechanism by $\beta$-phenylethyl isothiocyanate. Cancer Cell 10: 241-252. doi:10.1016/j.ccr.2006.08.009

Vera-Ramirez L, Sanchez-Rovira P, Ramirez-Tortosa CL, Quiles JL, Ramirez-Tortosa M, Lorente JA. 2013. Transcriptional shift identifies a set of genes driving breast cancer chemoresistance. PLoS One 8: e53983. doi:10.1371/journal.pone .0053983

Weeber F, Ooft SN, Dijkstra KK, Voest EE. 2017. Tumor organoids as a pre-clinical cancer model for drug discovery. Cell Chem Biol 24: 1092-1100. doi:10.1016/j.chembiol.2017.06 .012

Wu YM, Chen ZJ, Jiang GM, Zhang KS, Liu Q, Liang SW, Zhou Y, Huang HB, Du J, Wang HS. 2016. Inverse agonist of estrogenrelated receptor a suppresses the growth of triple negative breast cancer cells through ROS generation and interaction with multiple cell signaling pathways. Oncotarget 7: 1256812581.

Xia H, Dufour CR, Giguère V. 2019. ERRa as a bridge between transcription and function: role in liver metabolism and disease. Front Endocrinol 10: 206. doi:10.3389/fendo.2019.00206

Yang L, Moss T, Mangala LS, Marini J, Zhao H, Wahlig S, ArmaizPena G, Jiang D, Achreja A, Win J, et al. 2014. Metabolic shifts toward glutamine regulate tumor growth, invasion and bioenergetics in ovarian cancer. Mol Syst Biol 10: 728. doi:10.1002/ msb.20134892 


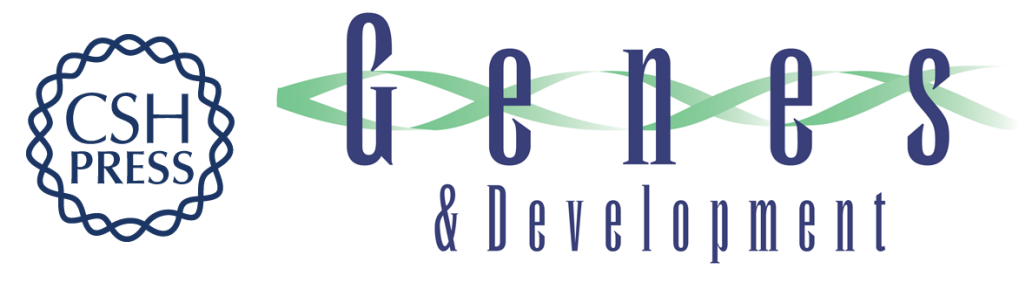

\title{
Estrogen-related receptors are targetable ROS sensors
}

Mathieu Vernier, Catherine R. Dufour, Shawn McGuirk, et al.

Genes Dev. 2020, 34: originally published online February 20, 2020

Access the most recent version at doi:10.1101/gad.330746.119

\begin{abstract}
Supplemental http://genesdev.cshlp.org/content/suppl/2020/02/20/gad.330746.119.DC1
Material

References This article cites 44 articles, 10 of which can be accessed free at:

http://genesdev.cshlp.org/content/34/7-8/544.full.html\#ref-list-1

Creative This article is distributed exclusively by Cold Spring Harbor Laboratory Press for the first Commons six months after the full-issue publication date (see

License http://genesdev.cshlp.org/site/misc/terms.xhtml). After six months, it is available under a Creative Commons License (Attribution-NonCommercial 4.0 International), as described at http://creativecommons.org/licenses/by-nc/4.0/.
\end{abstract}

Email Alerting Receive free email alerts when new articles cite this article - sign up in the box at the top Service right corner of the article or click here.

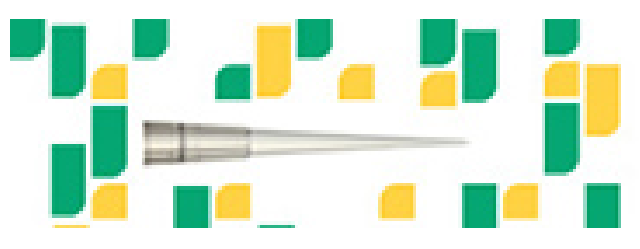

Focused on your science. 